Ann. Biol. anim. Bioch. Biophys., I964, 4 (4), 307-343.

\title{
RECHERCHES SUR LA MORPHOLOGIE ET LA TOPOGRAPHIE DE LA SUBSTANCE GRISE DANS LE BULBE RACHIDIEN DU BCEUF
}

\author{
R. BARONE et J. DOUCET \\ Laboratoire d'Anatomie, Ecole nationale vétérinaire, Lyon (Rhône)
}

SOMMAIRE

Les caractères morphologiques et structuraux de la substance grise, ainsi que la topographie de ses noyaux ont été étudiés sur les coupes sériées du bulbe rachidien d'une génisse de I 8 mois. Une reconstruction graphique des principaux noyaux a été réalisée.

On a noté le grand développement du noyau du nerf grand hypoglosse, du noyau intercalaire, du noyau dorsal du vague, du noyau ambigu, du noyau du facial, de l'olive protubérantielle et des noyaux du cordon latéral. Les noyaux gustatifs et salivaires, et certains noyaux vestibulaires sont mal définis. La jonction bulbo-protubérantielle est située plus oralement que chez la plupart des autres Mammifères, ce qui étend dans le même sens le territoire bulbaire.

Les Physiologistes et les Anatomistes ont consacré depuis quelques années de multiples recherches au tronc cérébral des Ruminants. Pour des raisons de commodité, celles-ci ont surtout porté sur le Mouton et la Chèvre. Bien des contrôles méritent pourtant d'être repris sur le Bœuf. Dans le cadre d'une expérimentation entreprise en ce sens, nous avons très vite éprouvé la nécessité d'une synthèse précise des données anatomiques. Les travaux existants sont en effet fragmentaires, épars et incomplets. Dans le double but de préparer des recherches de stéréotaxie et l'étude ultérieure fine des noyaux gris et des connexions, nous avons été amenés à reviser les différentes structures du tronc cérébral du Bœuf et leur topographie. L,e présent travail n'en constitue que la première partie. 


\section{MATÉRIEI, ET TECHNIQUE}

Cette étude est faite à partir du bulbe rachidien d'une génisse de 18 mois dont l'ensemble du tronc cérébral a été prélevé immédiatement après le sacrifice, après lavage au sérum physiologique par voie artérielle et fixation in situ au formol neutre à ro p. 1oo.

Le tronc cérébral et le premier segment cervical ont été débités en huit blocs qui ont été inclus à la paraffine et dont trois seulement ont été utilisés pour la présente étude (blocs $\mathrm{I}_{3} 85, \mathrm{I}_{3} 86, \mathrm{I}_{3} 87$ de la collection du Laboratoire).

Ceux-ci ont été débités en coupes histologiques sériées d'épaisseur uniforme de $5 \mu$ Une coupe sur vingt a été colorée par la technique de MARSLAND, GLEES et JiRIKSON et les coupes intermédiaires conservées pour des études ultérieures par d'autres techniques. Le total des coupes provenant des trois blocs et couvrant le territoire étudié pour la présente reconstruction s'est élevé à 4660 . La numération a été établie d'arrière en avant, c'est-à-dire du collet du bulbe (I) au bord caudal de la protubérance annulaire (4660). La coupe numéro I correspond, en réalité dans la collection, à la lame 400 au bloc I385. La coupe 4660 correspond à la lame 400 du bloc I387. Enfin il nous paraît utile de préciser que la pointe du calamus est intéressée par la coupe i 240 de la présente numération.

\section{RÉSULTATS}

Nous nous bornerons à l'exposé des caractères morphologiques essentiels et de la topographie des noyaux gris, réservant pour des publications ultérienres l'étude de la structure fine et des connexions.

Nous commencerons par les noyaux appartenant aux six dernières paires des nerfs crâniens, que nous classerons par colonnes. Nous décrirons ensuite les formations grises n'appartenant pas à ces colonnes.

La topographie sera résumée par une suite de dix photographies numérotées de I à Io d'arrière en avant, et montrant des sections transversales du bulbe à des niveaux sensiblement équidistants, et đeux planches dessinées, résumant la disposition cles diverses colonnes. Quelques photomicrographies illustreront en outre les types structuraux les plus caractéristiques des principaux noyaux.

\section{I. - Colonne extéroceptive}

Elle comporte le noyau gélatineux du trijumeau et les noyaux acoustiques.

\section{Io Noyau gélatineux.}

Très volumineux, ce noyau commence oralement au voisinage même de l'émergence du trijumeau et s'étend en arrière jusqu'au premier segment de la moelle cervicale et au-delà. I1 est toujours aisément reconnaissable, sur les coupes transversales, à sa situation médiale par rapport à la racine descendante du trijumeau, qui dessine un épais croissant à son bord latérodorsal.

Il est aussi reconnaissable à sa structure particulière, tout à fait comparable à celle de la substance de Rolando, qui coiffe la corne dorsale de la moelle et dont il continue d'ailleurs manifestement le système. Indépendamment des cellules névrogliques, on y retrouve les deux types cellulaires caractéristiques de cette substance : I $^{0}$ de très petites cellules à corps ovoïde ou piriforme, qui nous semblent représenter les cellules de GIERKE, out les cellules limitrophes décrites par CAJAI, dans la moelle épinière $; 2^{\circ}$ des cellules fusiformes ou étoilées, le plus souvent plus grandes que les 
précédentes, et dont certaines deviennent beaucoup plus volumineuses dans les régions les plus orales du noyau. La substance de ce noyau est en outre finement subdivisée par les passages de groupes fibrillaires les uns longitudinaux, d'autres radiaires ou transversaux. Les premières de ces fibres constituent une mince couche à la surface et d'autre part un feutrage dans la profondeur du noyau et à sa partie médiale. Les fibres radiaires et les fibres transversales sont particulièrement visibles sur les coupes transversales.

Ce noyau, déjà parfaitement discernable dans le premier segment cervical, c'est-à-dire en dessous même du niveau initial de notre reconstruction, peut être suivi en direction orale jusqu'à la coupe 4400 , c'est-à-dire tout près du bord caudal de la protubérance annulaire. Dans la région post-calamique du bulbe comme dans la moelle épinière, il nous a paru nettement plus volumineux que chez le Cheval, mais moins subdivisé et mieux délimité. Sa surface de section est importante dans la moitié caudale du bulbe. Fille se rétrécit assez nettement en regard de l'émergence du glosso-pharyngien et diminue ensuite peu à peu dans la moitié orale du bulbe. Sur les coupes situées oralement au $\mathrm{n}^{\mathrm{s}} 4000$, il est réduit à des îlots épars et fragmenté du côté médial par les fibres de la racine motrice du trijumeau. Notre planche $n^{\circ}$ I I ne donne pas une idée exacte de cette réduction car elle figure la projection orthogonale des bords extrêmes de tous ces groupes cellulaires.

Cette extrémité du noyau gélatineux se met en contact latéralement et dorsalement avec le noyau sensitif pontin dont nous parlerons plus loin.

\section{$2^{\circ}$ Tubercule acoustique ou noyau acoustique dorsal.}

Ce noyau est situé dans la moitié orale du bulbe. Son extrémité caudale surmonte dorsalement et en dehors le corps restiforme. Sa partie orale devient de plus en plus latérale et s'accole largement au noyau acoustique ventral à la base de la racine du nerf acoustique.

Le tubercule acoustique est formé de cellules polymorphes plus ou moins sphériques, de petite taille. Il est nettement divisible en deux zones ; 1'une, superficielle, la seule représentée à son extrémité orale où elle vient recouvrir le noyau ventral, l'autre, profonde. Dans la première, les cellules sont moins nombreuses mais certaines d'entre elles, relativement volumineuses, prennent la forme multipolaire ou bipolaire à grand axe généralement radiaire. L,es fibres de cette zone sont très fines, pour la plupart amyéliniques ou peu myélinisées. La partie profonde du noyau comporte de façon assez uniforme un riche feutrage de fibres plus volumineuses et de cellules petites et rondes. Ces dernières deviennent nettement plus nombreuses au contact de la portion superficielle où leur aspect et leur disposition évoquent presque en certains points celui des grains du cervelet.

Suivi d'arrière en avant, ce noyau apparaît sur la coupe 2400 et s'étend oralement jusqu'à la coupe 37 Io. Toujours étalé à la surface bulbaire, au bord dorso-latéral des coupes, il apparaît d'abord sous la forme d'une bande étroite. Il augmente de taille jusqu'au niveau de la coupe 2880 et s'amincit ensuite progressivement dans sa partie orale.

\section{$3^{\circ}$ Noyau acoustique ventral.}

Placé ventralement au précédent, qu'il rejoint à son extrémité orale, ce noyau s'étend beaucoup moins en direction caudale. 
Il est caractérisé par le grand nombre de fibres de gros calibre et bien myélinisées, qui lui servent littéralement de trame et dont certaines le quittent en direction de l'olive protubérantielle. Ses cellules sont de taille moyenne, mais dans l'ensemble nettement plus grandes que celles du noyau précédent, généralement arrondies ou piriformes. Elles constituent deux groupes assez distincts : 1'un dorsal, plus spécialement uni au groupe profond du tubercule acoustique et l'autre ventral où les cellules sont moins nombrẹses, séparées par d'importants paquets de fibres mieux myélinisées.

Les limites extrêmes de ce noyau s'inscrivent entre les coupes 2920 et 3980. L'extrémité caudale se trouve donc en regard de la moitié du tubercule acoustique. Sur les coupes transversales, le noyau ventral est engagé comme un coin à pointe dorso-médiale entre le corps restiforme et le noyau dorsal ou tubercule acoustique. Les deux noyaux disparaissent d'ailleurs oralement presque en même temps.

Comme WeLENTO, nous avons trouvé, à différents niveaux entre les fibres du nerf acoustique lui-même, des groupes de cellules qui ne se rattachent ni au noyau acoustique ventral ni au noyau acoustique dorsal. Ces cellules multipolaires, de taille moyenne, ont déjà été décrites par Martin, chez le Chat. Nous en ignorons la signification fonctionnelle.

\section{II. - Colonne proprioceptive}

Elle comprend fondamentalement dans le bulbe l'ensemble des noyaux vestibulaires, mais elle se poursuit dans la protubérance et le mésencéphale par des noyaux du trijumeau (noyau sensitif pontin et noyau mésencéphalique) que nous ne ferons que citer ici.

\section{Io Noyau vestibulaire descendant.}

C'est un noyau important, qui peut être suivi sur presque toute la longueur du bulbe. WELENTO en a déjà donné une description minutieuse, dont nous n'avons d'ailleurs pas retrouvé tous les détails. Il est accompagné sur toute sa longueur par la racine vestibulaire descendante dont les multiples faisceaux découpent sa substance autant qu'ils la bordent. Il est mal délimité, très fragmenté en multiples petits noyaux secondaires. Il est découpé par de nombreux passages de fibres radiaires ou transversales et surtout d'importants faisceaux longitudinaux bien myélinisés.

Les cellules sont multipolaires, de taille moyenne ou légèrement grande, pour la plupart ovoïdes ou piriformes, plus rarement étoilées.

Dans sa partie caudale, ce noyau constitue une masse irrégulièrement ovale à grand axe transversal. Un peu oralement au niveau du calamus, il se subdivise en trois groupes de cellules, médial, intermédiaire et latéral, qui s'unissent entre eux de façon irrégulière en certains points. Un quatrième groupe s'individualise un peu plus en avant en situation plus ventrale. Plus en avant encore, cette disposition devient indiscernable.

Son extrémité orale commence à apparaître un peu caudalement à la pénétration du nerf stato-acoustique dans le bulbe et à l'individualisation de la racine descendante du nerf vestibulaire, soit au voisinage de la coupe 3900 . Son volume s'accroît rapidement en direction caudale puis décroît progressive et se réduit à partir de la coupe 600 à quelques fragments épars qui deviennent indiscernables à la coupe 460 . 
Son extrémité orale est située au bord ventro-latéral du noyau triangulaire ou descendant médial et ventro-médialement au noyau de DeiTERs. Il est, dans tout son trajet, caractérisé par sa situation dorsale puis dorso-médiale par rapport au noyau gélatineux. Son extrémité caudale rejoint le noyau de von Monakow en se plaçant dorso-latéralement à ce dernier, dont il devient difficile à délimiter.

\section{$2^{\circ}$ Noyau vestibulaire latéral ou de DeITERS}

Situé à la partie dorso-latérale et orale du bulbe, en avant du corps restiforme, ce noyau est composé de cellules caractéristiques, sphéroïdales, uni- ou bipolaires, grandes et moyennes, qui augmentent de taille dans la portion orale. Selon WELENTO, ces cellules constituent caudalement trois grands groupes, fusionnés oralement en une masse unique étendue entre le noyau moteur oculaire externe et le corps restiforme. Cette disposition ne nous a pas paru évidente. Par contre, il existe une réelle intrication avec l'extrémité orale du noyau vestibulaire descendant.

Relativement court mais large, ce noyau s'étend entre les niveaux marqués par les coupes I 920 et 3870 . Son extrémité orale est logée entre les extrémités correspondantes du noyau de BECHTEREw, qui lui est dorso-latéral, et du noyau moteur oculaire externe. L'extrémité caudale se trouve comprise entre le corps restiforme, le noyau vestibulaire descendant et la racine du trijumeau, dans un plan un peu oral à la limite caudale du noyau triangulaire.

\section{$3^{\circ}$ Noyau triangulaire ou noyau descendant interne du nerf vestibulaire.}

Chez les Bovins, ce noyau représente en fait non seulement le noyau triangulaire proprement dit des autres espèces, mais encore le noyau médial de Scrwalbe, qui ne peut en rien être distingué dans la région orale.

Il est en outre caractérisé par sa situation voisine du plancher du quatrième ventricule et par l'abondance de ses fibres, relativement minces et peu myélinisées. I1 est formé de cellules petites ou moyennes, triangulaires ou multipolaires.

Plus étiré que le précédent, ce noyau s'étend entre les limites fournies par les coupes 1500 et 3700 . Son extrémité orale se place sur un plan un peu plus caudal que celle du noyau vestibulaire descendant. Fille est étalée un peu en arrière et en dedans de l'angle latéral du IVe ventricule, médialement et dorsalement au noyau vestibulaire latéral (DEITERS), puis dorsalement au noyau vestibulaire descendant. Plus en arrière, ce noyau s'élargit, devient médial au noyau vestibulaire descendant et prend sur des coupes transversales une forme triangulaire curviligne. Son extrémité caudale se place entre le noyau vestibulaire descendant et les noyaux cardio-pneumo-entérique et du faisceau solitaire, un peu en dehors de l'area postrema et dorsalement au noyau de von MoNAKow. Le noyau triangulaire occupe sensiblement, sur le plancher du IVe ventricule, la place de l'aile blanche externe.

$4^{\circ}$ Noyau vestibulaire supérieur ou de BECHTEREW.

Il s'agit d'un noyau peu volumineux, constitué de cellules sphéroïdales ou étoilées, de taille petite ou moyenne. Les fibres, particulièrement nombreuses dans la partie moyenne, permettent de discerner deux groupes cellulaires, triangulaires sur les coupes et fusionnés oralement.

Ce noyau est situé dorso-latéralement au noyau de DeITERs, en dehors de l'angle latéral du IVe ventricule et à la base du pédoncule cérébelleux postérieur. 
Nous le rencontrons sur les coupes de 3360 à 4000 . Sa limite caudale se situe sensiblement sur le même plan transversal que celui du noyau moteur oculaire externe. L'extrémité orale se place sous le pédoncule cérébelleux antérieur, sur un plan voisin de la limite caudale du noyau mésencéphalique du trijumeau.

$5^{\circ}$ Noyau sensitif pontin.

Ce noyau n'est cité ici que pour mémoire car il appartient à la protubérance annulaire et seule sa partie caudale est intéressée par les coupes que nous avons étudiées. Il est placé à l'extrémité orale du noyau gélatineux, dans une position dorsolatérale à celle-ci. Il est formé de cellules multipolaires et fusiformes de petite taille, et de quelques petites cellules rondes au voisinage de la racine motrice du trijumeau.

Il est situé entre les fibres de cette dernière racine, du côté médial, et la racine mésencéphalique du trijumeau du côté latéral.

A l'extréme limite orale du bulbe commence en outre l'important noyau mésencéphalique du trijumeau, que nous ne faisons également que citer, Ce noyau est placé en position tout à fait dorsale, au contact même đut pédoncule cérébelleux antérieur qui l'accompagnera sur un long parcours dans le mésencéphale.

\section{III. - Colonne viscéroceptive}

Cette colonne est essentiellement constituée par le noyau du faisceau solitaire, dont on peut considérer comme des différenciations les noyaux gustatifs.

\section{Io Noyau du faisceau solitaire.}

Nous n'avons trouvé aucune description de ce noyau chez le Bouf dans la littérature. Toujours caractérisé par son contact avec le faisceau solitaire et sa situation voisine du noyau dorsal du pneumogastrique (cardio-pneumo-entérique), par rapport auquel il est dorso-latéral, ce noyau s'étend sur les deux tiers caudaux du bulbe rachidien.

Sa structure n'est pas sans analogie avec celle du noyau gélatineux. Il montre en effet, au sein d'un feutrage fibrillaire dense, des cellules nerveuses de taille petite ou très petite. Celles-ci, très polymorphes, n'ont pas d'arrangement régulier. Elles sont souvent ovoïdes et fusiformes, parfois triangulaires, tendant même à 1'aspect multipolaire. Toutefois, on , ne trouve nulle part de cellules multipolaires de taille moyenne comme en présente le noyau gélatineux.

Le noyau du faisceau solitaire constitue en réalité un véritable complexe, dans lequel plusieurs parties peuvent être distinguées. La principale longe sur toute sa longueur le bord médial du faisceau solitaire qui, toujours aisément repérable sur les coupes, permet de la situer sans hésitation. Elle équiyaut au " ganglion descendant "décrit par CAJAI, chez la Souris et chez le Chat. C'est une colonne grise qui commence un peu oralement par rapport au noyau dorsal du pneumogastrique et descend jusque dans le premier segment de la moelle cervicale. Son extrémité orale est située au voisinage de la coupe $275^{\circ}$, c'est-à-dire au niveau de 1 'implantation des raçines moyennes du nerf glossopharyngien. Ellle se met à ce niveau en continuité avec le noyau gustatif caudal et ses limites sont très difficiles à préciser.

Un peu plus caudalement, cette portion médiale du faisceau solitaire devient mieux discernable. Sa section, relativement large, devient ovoïde à grand, axe trans- 
versal et, dès le niveau de la coupe 2500 , prend sa position caractéristique entre le faisceau solitaire, lui aussi mieux délimité et qui lui est latéral, et le noyau dorsal du pneumogastrique, qui lui est ventro-médial. Sur les coupes plus caudales (2300 à I900) il présente une coupe presque circulaire puis, à partir du niveau I800, s'étire transversalement. Entre ce dernier niveau et le voisinage de la coupe rooo, il est très étiré en travers, aminci du côté médial, où il est mal délimité, tandis que du côté latéral il s'élargit, reste bien délimité et semble se bifurquer pour venir enserrer ventralement et dorsalement le faisceau solitaire. Plus bas encore, son volume se réduit. Il se rapproche peu à peu du plan médian et devient franchement dorsal au noyau cardio-pneumo-entérique. Il est alors très mal défini, surtout du côté médial. En effet, caudalement au calamus, les deux noyaux, droit et gauche, sont unis dorsalement au canal de l'épendyme par une traînée diffuse de substance grise qui constitue l'équivalent du "ganglion commissural " de CAJAI.

La dernière partie du complexe gris qui nous occupe représente le " ganglion interstitiel ou latéral " de CAJAL. C'est une colonne grise beaucoup plus grêle et plus courte que la précédente, et qui n'est discernable que dans les coupes comprises entre les $\mathbf{n}^{0}$ I Ioo et I60o. De section ovalaire, elle paraît enclavée dans le bord latérodorsal du faisceau solitaire, dorsalement auquel elle s'unit en quelques points avec la colonne médiale. Ce noyau latéral semble surtout recevoir les fibres d'un petit faisceau blanc longitudinal situé à son bord médial et qui nous paraît devoir être considéré comme une partie détachée du faisceau solitaire proprement dit. Il possède probablement une signification fonctionnelle propre, qui reste à préciser.

\section{$\left.2^{\circ}\right)$ Noyaux gustatifs.}

Il s'agit en réalité d'un seul noyau (Noyau gustatif de Nageotte), mal délimité à certains niveaux, étendu sur le tiers oral du bulbe rachidien dans une position qui prolonge celle du noyau faisceau solitaire, ventralement au système vestibulaire et dorso-médialement au noyau gélatineux du trijumeau. I1 reçoit dans sa partie orale les fibres du nerf intermédiaire de Wrisberg et dans sa partie caudale les fibres les plus antérieures du glosso-pharyngien. La distinction entre un noyau gustatif oral, recevant le nerf de Wrisberg, et un noyau gustatif caudal, connecté au glosso-pharyngien, nous paraît toute théorique.

La texture est tout à fait comparable à celle du noyau du faisceau solitaire, avec lequel s'établit une continuité. Les cellules ont toutefois une taille un peu plus grande et une forme plus souvent sphéroïdale ou piriforme.

Le noyau gustatif s'étend du voisinage de la coupe 2800 à celui de la coupe 4 I5O. Sur toute son étendue, il reste à proximité du bord dorso-médial de la racine descendante du trijumeau. Son extrémité orale se place ventralement à la racine mésencéphalique et au noyau sensitif pontin de ce nerf.

Dans ses deux tiers caudaux, il est relativement épais, mais fort difficile à délimiter. La portion du faisceau solitaire qui lui correspond et longe en principe son bord latéral est elle-même mal délimitée sur les coupes transversales. Elle est fragmentée en multiples fascicules mêlés à la substance grise et, pour certains, en contact avec la racine descendante du trijumeau.

Le tiers oral du noyau gustatif redevient bien délimité. Il est en outre subdivisé en deux colonnes grises parallèles, l'une ventrale et l'autre dorsale, qui finissent par se réunir. Le faisceaut blanc qui les accompagne est lui-même mieux distinct. Il groupe 
alors les fibres de l'intermédiaire de Wrisberg et peut-être quelques faisceaux de fibres trigéminées.

\section{IV. - Colonne viscéro-efférente}

Cette colonne comporte le noyau dorsal du pneumogastrique et, plus oralement, les noyaux salivaires, puis le noyau lacrymo-muco-nasal.

\section{Io) Noyau dorsal du pneumogastrique.}

Ce noyau mérite encore le nom de cardio-pneumo-entérique, en raison de la destination dez fibres qui en proviennent. Toutefois, son extrémité orale fournit une petite partie des fibres efférentes du glosso-pharyngien, probablement le contingent vaso-moteur.

Il est toujours aisément reconnaissable au caractère de ses cellules, remarquablement uniforme à tous les niveaux. Il s'agit de cellules de taille moyenne et d'aspect le plus souvent fusiforme, plus rarement triangulaire ou multipolaire. Elles sont orientées parallèlement les unes aux autres en un semis relativement dense. Dans la partie caudale et la partie moyenne du noyau, leur orientation est sensiblement transversale, ce qui leur donne un aspect caractéristique sur les coupes. Leur orientation devient oblique, puis presque longitudinale dans la partie la plus orale, cê qui tend à leur donner un aspect plus ou moins rond. Aucune division ne se manifeste dans ce noyau, si ce n'est dans la région tout à fait orale une tendance à une scission en deux groupes, l'un dorso-médial et l'autre ventro-latéral.

Le noyau dorsal du vague est déjà distinct dans le premier segment de la moelle cervicale. Il se renforce très progressivement en accompagnant dorsalement et latéralement le noyau du nerf hypoglosse, au-delà duquel il se continue oralement pour se terminer au niveau de la coupe 2420 . Sa partie caudale se présente comme une bande aplatie dorso-ventralement et située latéralement à la région épendymaire, au-dessus de laquelle elle vient jusqu'au voisinage immédiat du plan médian. A partir de la région calamique, le noyau dorsal du vague se trouve reporté plus latéralement. comme celui $d u$ faisceau solitaire qui l'accompagne en dehors sur toute sa longueur, Il devient alors nettement plus volumineux et ovalaire ou arrondi sur les coupes transversales. Il est placé au voisinage du plancher du IVe ventricule, médialement au sulcus simitans où il constitue la base de l'aile grise. Son extrémité orale s'éloigne de ce plancher en devenant plus profonde et plus latérale. Elle est située à peu prè; à mi-longueur du bulbe rachidien où elle se termine assez brusquement.

\section{0) Noyaux salivaires.}

La littérature est très pauvre sur ces noyaux, qui sont souvent mentionnés mais presque jamais décrits. Les seules indications un peu plus précises sont données par Pat'ison et Holman à propos du bulbe du Mouton. En voici les termes :

Salivaire inférieur : juste au-devant de l'extrémité antérieure du noyau du facial, et dans une position légèrement plus dorsale, un petit groupe de cellules semblables à celles du noyau ambigu apparaît brusquement, persiste sur quelques coupes et disparaît ensuite tout aussi brusquement. Ce groupe cellulaire correspond, par la dimension et la situation, au noyau salivaire inférieur décrit chez le Chien.

Salivaire supérieur : ce noyau est tout à fait semblable en volume et situation au noyau salivaire inférieur, si ce n'est qu'il est plus court et apparaît à un niveau 
un peu plus rostral. Il commence et finit brusquement et est formé de cellules comparables à celles du noyau ambigu.

Nous avons bien retrouvé chez la Vache deux groupements cellulaires répondant aux descriptions qui précèdent. Le premier, le plus caudal, s'étend sur le court espace compris entre les coupes 3800 et 3900 . Formé de cellules multipolaires de taille moyenne, il est placé dorsalement à l'extrémité orale du noyau du facial, sous la partie descendante de ce nerf, latéro-ventralement et à quelque distance du noyau oculomoteur. L'autre groupe cellulaire, plus oral et guère mieux développé, s'étend entre les coupes 4020 et 4 IOO. Il présente des caractères analogues et se place contre la portion descendante du facial.

Il nous paraît pourtant difficile d'admettre comme une certitude qu'il s'agisse là des deux noyaux"salivaires. En premier lieu, cette topographie pourrait être admissible pour un noyau salivaire oral mais elle ne l'est pas pour un noyau salivaire caudal, dont les fibres devraient sortir avec celles du glosso-pharyngien. D'autre part, la très faible étendue de ces groupes cellulaires est mal compatible avec l'énorme volume de la secrétion salivaire chez les Bovins. Enfin, lorsqu'on examine avec soin le trajet intrabulbaire du facial en remontant depuis son émergence, on voit nettement des faisceaux de fibres le quitter à angle très aigu en direction dorso-médiale vers le bord latéral du plancher ventriculaire. I a partie la plus orale de cet éventail de fibres semble bien provenir du noyau lacrymo-muco-nasal; la plus postérieure nous paraît appartenir au noyau salivaire oral.

$\mathrm{Si}$ on admet cette interprétation - - que nous présentons avec toutes les réserves qu'appelle l'absence d'un contrôle expérimental - les groupes cellulaires décrits ci-dessus pourraient être considérés comme des dépendances du noyau oculo-moteur externe (ou de la substance réticulée) et les noyaux salivaires cherchés plus dorsomédialement, près du plancher ventriculaire. Un semis cellulaire existe en effet à ce niveau, mais il est fort mal défini et ne montre pas de subdivision nette en deux noyaux, oral et caudal. Comme pour le noyau gustatif, il nous parait plus conforme à la réalité de décrire un seul noyau salivaire, dont la division reste assez conventionnelle dans 1'espèce bovine.

Ce noyau apparaît médialement à l'extrémité orale du noyau dorsal du pneumogastrique. Il s'étend ensuite oralement à ce dernier noyau en s'élargissant. Étalé à faible distance du plancher du IVe ventricule, il est beaucoup moins bien caractérisé et délimité que le noyau dorsal du vague. De nombreuses fibres l'unissent au noyau du faisceau solitaire et au noyau gustatif. Ses cellules sont polymorphes et toujours petites, pour la plupart multipolaires ou triangulaires. D'autres, éparses, sont fusiformes, très argyrophiles, tout à fait analogues à celles du noyau dorsal du vague. La limite caudale est voisine de la coupe 2200 . Elle semble prolonger le nucleus praepositus hypoglossi et se place entre celui-ci et l'extrémité orale du noyau dorsal du pneumogastrique.

Le noyau salivaire s'élargit ensuite jusqu'au niveau de la coupe 2800 , puis se rétrécit à nouveau et s'effile longuement jusqu'au contact du genou du nerf facial. Son calibre est déjà faible sur la coupe 3 roo mais on peut retrouver son prolongement jusqu'au voisinage de la coupe 3500, dorsalement au genou du facial.

\section{$\left.3^{\circ}\right)$ Noyau lacrymo-muco-nasal.}

Relativement étendu, mais plus mal défini encore, ce noyau, étiré latéralement au bord du plancher ventriculaire, prolonge oro-dorsalement le précédent. Il apparaît 
dorsalement au genou du facial et au noyau du nerf oculo-moteur externe et se prolonge oralement jusqu'au voisinage du noyau du locus coeruleus et de ses énormes. cellules pigmentaires. Sa partie orale est en contact médialement avec les noyaux. tegmentaires du pont.

Ce noyau organique du facial est donc placé à la limite du bulbe rachidien et. de la protubérance, mais sa majeure partie appartient topographiquement au bulbe.

\section{V. - Colonne somito-motrice}

A cette colonne appartiennent, dans le bulbe : le noyau du nerf grand hypoglosse, ses noyaux connexes (noyau intercalaire et nucleus praepositus) et plus oralement, le noyau du nerf oculo-moteur externe.

\section{ro) Noyau du nerf grand hypoglosse.}

Ce noyaut est fort développé chez le Bouf, peut-être en raison de la très grande mobilité de la langue, employée dans cette espèce pour la préhension des. aliments. Il est toutefois moins étendu oralement que chez les Équidès. Il s'agit en réalité non pas d'un noyau unique, mais d'un complexe comprenant deux groupes cellulaires principaux, 1'un dorsa1, volumineux, l'autre ventral, et d'un groupe accessoire latéro-ventral.

a) Noyau dorsal. - C'est le plus étendu. Il s'étire sensiblement sur la moitié caudale du bulbe, au voisinage immédiat du plancher du quatrième ventricule où il correspond, près du sillon médian, à la saillie de l'aile blanche interne. Il est caractérisé par ses cellules étoilées, de taille moyenne ou grande, qui constituent des sousgroupes : l'un médial, voisin du canal épendymaire dans la partie post-calamique dtı bulbe, le second intermédiaire et le dernier dorso-latéral. Comme l'indique CHomiak, cette subdivision est particulièrement nette vers la mi-longueur du noyau.

Ce noyau dorsal commence au niveau même du collet du bulbe, très près du plan médian. Il s'étend un peu latéralement dans sa portion précalamique et se termine assez brusquement au voisinage de la coupe 2300 .

b) Noyau ventral. - Situé ventralement, mais aussi un peu latéralement par rapport au précédent, ce noyau est moins bien délimité et s'étenđ moins loin en direction orale. Il est constitué de cellules multipolaires de taille moyenne, moins colorables que celles du noyau dorsal et analogues à celles de la substance réticulée. Il est situé au contact même des faisceaux de fibres du nerf hypoglosse et surtout développé médialement à ceux-ci. Nous 1'avons rencontré depuis le niveau du collet du bulbe jusqu'au voisinage de la coupe I 880 .

c) Noyau accessoire. - Il a la même texture que le précédent et se trouve placé latéralement au parcours des fibres du nerf grand hypoglosse. Il est peu distinct des éléments réticulaires qui l'entourent.

\section{$\left.2^{\circ}\right)$ Noyau intercalaire de Staḋerini.}

C'est, chez le Boeuf, un noyau important dont le développement est peut-être en relation avec la grande mobilité de la langue. Il est en effet uni par de nombreuses fibres au noyau de l'hypoglosse ainsi qu'aux noyaux sensitifs. On peut supposer qu'il joue un rôle important dans la coordination des mouvements de la langue. 'Tou- 
tefois, comme les noyaux eux-mêmes de l'hypoglosse, il a une situation générale plus caudale que chez les Équidés.

Il est formé de cellules multipolaires de taille moyenne ou petite, nettement inférieure à celles du noyau dorsal de l'hypoglosse. Il constitue une colonne ovalaire ou circulaire sur les coupes, toujours située dorso-latéralement à ce dernier noyau et ventralement au noyau cardio-pneumo-entérique. Nous avons trouvé sa limite caudale at niveau de la coupe 440 , tandis que sa limite orale ne dépasse pas la coupe 2000.

$\left.3^{\circ}\right)$ Nucleus praepositus hypoglossi.

Ce noyau semble prolonger oralement le noyau dorsal de l'hypoglosse. Il est formé de cellules multipolaires ou rondes, de taille moyenne ou petite. Son extrémité caudale est plus ou moins confondue avec le noyau dorsal de l'hypoglosse. Son extrémité orale s'étire jusqu'à la coupe 2580 , en se plaçant médialement au noyau salivaire. Son développement, relativement grand chez le Bœuf, et ses connexions avec le noyau salivaire peuvent laisser supposer qu'il intervient pour coordonner, dans une certaine mesure, la sécrétion salivaire et les mouvements de la langue.

\section{$\left.4^{\circ}\right)$ Noyau du nerf moteur oculaire externe.}

Ce noyau, situé dans la partie orale du bulbe, est voisin du plancher du quatrième ventricule. Il est placé ventralement au genou du nerf facial, dans la concavité duquel il est littéralement moulé. Il est formé de cellules multipolaires de taille relativement grande, constituant un agrégat de forme ovalaire, dont le grand axe est presque vertical, à peine oblique en bas et en dehors. Sa limite caudale, portée par la coupe 330o, est sur un plan transversal qui coupe le noyau du nerf facial un peu au-dessus de son quart antérieur. L'extrémité orale se trouve sur la coupe 3960. CHomiak divise ce noyau en deux groupes dont la délimitation ne nous a pas paru vraiment caractéristique.

Un examen attentif permet de repérer un noyau accessoire très court, formé d'un petit groupe de cellules multipolaires, à mi-distance entre le noyau principal et l'olive protubérantielle. Ce noyau accessoire commanderait le muscle choanoïde.

\section{VI. - Colonne branchio-motrice}

Cette colonne comporte trois importants noyaux noteurs : le noyau ambigu, le noyau du nerf facial et le noyau masticateur. Nous y rattacherons les noyaux bulbaires du nerf spinal dont la véritable nature paraît encore mal établie.

\section{I') Noyau bulbaire du nerf spinal.}

La signification fonctionnelle du spinal bulbaire n'est pas encore clairement établie. On s'accorde en général pour considérer les cellules d'origine de cette portion du spinal comme dérivées du noyau dorsal du pneumogastrique. Quelques auteurs estiment pourtant qu'il s'agit d'un groupe cellulaire différencié de la partie caudale du noyau ambigu.

Chez le Bœuf comme chez le Cheval, Chomiak distingue deux noyaux d'origine du spinal bulbaire, l'un dorsal et l'autre ventral. Le noyau dorsal, ou mieux dorsomédial, placé au bord latéral de la partie caudale du noyau cardio-pneumo-entérique, 
nous paraît beaucoup trop mal différencié de ce dernier, avec lequel il se confond d'ailleurs en plusieurs points, pour être rattaché avec certitude au nerf spinal. Il s'agit plutôt d'une différenciation dans le noyau dorsal du vague. Le noyau ventral nous paraît donc être le seul appartenant réellement au spinal bulbaire.

Il est situé beaucoup plus latéralement et ventralement, mais sa morphologie évoque clairement une dérivation du noyau dorsal du vague, Il est en effet formé de cellules fusiformes exactement comparables à celles de ce dernier, mais toutefois plus grandes. Ces cellules, rangées presque parallèlement les unes aux autres, ont leur grand axe sensiblement placé dans le plan des coupes transversales et orienté de telle sorte que l'un des pôles se prolonge manifestement vers le noyau dorsal du vague et l'autre s'étire ventro-latéralement vers l'émergence radicu laire. Placé médio-ventralement au noyau gélatineux, le noyau du spinal commence à apparaître au niveau de la coupe r5o. Lorsqu'on le suit sur des coupes de plus en plus orales, on constate qu'il s'élargit et que ses cellules prennent progressivement un aspect irrégulier puis multipolaire, de sorte que la transition s'établit insensiblement avec le noyau ambigu. Il nous semble que la limite entre les deux formations peut être située aux environs de la coupe $75^{\circ}$.

\section{$\left.2^{\circ}\right)$ Noyau ambigu.}

Ce noyau est mal délimité. Il s'étend à travers la substance réticulée approximativement sur le tiers caudal du bulbe. C'est un ensemble de grandes cellules multipolaires, parfois allongées dans le sens des fibres radiculaires qui en procèdent. Son développement est remarquable chez les Ruminants et l'on peut penser qu'il est en relation avec l'épaisseur des couches musculaires de l'œsophage dans ces espèces. Toutefois, il est difficile de distinguer en son sein des groupements cellulaires particuliers et même d'en préciser les limites en raison du développement de la substance réticulée à son voisinage.

Le noyau ambigu est placé ventro-médialement au noyau gélatineux et ventrolatéralement au noyau du nerf grand hypoglosse. Il est continué en arrière par le noyau du spinal bulbaire aux environs de la coupe $75^{\circ}$. D'abord relativement large, il se rétrécit oralement en devenant un peu plus latéral et peut être suivi jusqu'au voisinage de la coupe rgoo.

\section{$\left.3^{\circ}\right)$ Noyau du nerf facial.}

Ce noyau, volumineux et bien différencié, s'étend sur près des deux tiers oraux du bulbe rachidien, depuis le niveau du pôle oral de l'olive inférieure jusqu'à celui de l'olive supérieure. Il est caractérisé par ses cellules multipolaires de taille moyenne ou plus généralement grande et par sa situation médio-ventrale par rapport au noyau gélatineux.

Sa systématisation est relativement complexe mais, surtout dans ses parties moyenne et orale, il est aisé de reconnaître les grandes divisions fondamentales déjà décrites dans les autres Mammifères.

Son extrémité caudale, grêle et comme effilée, apparaît sensiblement en regard du pôle oral de l'olive bulbaire, soit au voisinage de la coupe I8oo. Suivi sur des coupes de plus en plus orales, le noyau n'augmente que très progressivement de volume. Il présente en outre un aspect lobé, ou plutôt moniliforme, faisant alterner des parties élargies et de forts étranglements. Toutefois, très rapidement apparaît (niveau 
Igoo) une division fondamentale en un noyau dorsal et un noyau ventral. Le premier est d'abord dorso-latéral et le plus volumineux. Les deux noyaux sont plus ou moins distincts selon les niveaux, indiscernables dans les parties étranglées. Puis le noyau ventral devient plus volumineux, tandis que le dorsal s'amincit et s'étire transversalement. Au niveau de la coupe $275^{\circ}$ apparaît un nouveau noyau qui semble résulter du dédoublement du noyau ventral. C'est un noyau médial ou médio-ventral, qui ne tarde pas à devenir volumineux. 'Toujours bien distinct et nettement isolé, ce nouveaui noyau est en outre caractérisé par ses cellules multipolaires de taille plus petite que celles de son voisin. Presque au même niveau commence à se distinguer un noyau latéral, moins distinct, en dehors du noyau médial.

Dès le niveau 2800 est ainsi réalisée la disposition typique, déjà bien décrite dans beaucoup d'autres Mammifères. Le noyau dorsal, qui tend lui-même à se diviser en deux sous-groupes, médial et latéral, restera réduit mais pourra être suivi jusque très près de la terminaison orale du noyau du facial. Il commanderait les muscles peaucier $d u$ front et orbiculaire des paupières. Le groupe des noyaux ventraux, beaucoup plus développé, est lui-même divisible en trois noyaux. Le médial, bien distinct jusqu'au niveau de la coupe $365^{\circ}$, où il disparaît, serait affecté à la musculature de l'oreille (moyenne et surtout externe). Il est en effet senl exempt de chromatolyse lorsque le facial est coupé, sur des animaux d'expérience, après l'émission des branches auriculaires. Son grand développement chez le Bœuf est peut-être lié à celui des muscles peauciers de l'oreille. Le groupe intermédiaire, de loin le plus important et qui représente seul le noyau du facial à son extrémité orale, commanderait la musculature de la face. Le groupe latéral, le plus faible et le moins net, est un peu mieux développé oralement, ou il tend à devenir dorsal. Sa signification n'est pas établie ; peut-être anime-t-il le digastrique (justement faible chez le Bouf) et les muscles hyoïdiens supérieurs?

Le noyau du facial, dont 1'épaisseur est maxima dans son tiers oral, disparaît assez brusquement, au voisinage de la coupe 3900 , à peu près en regard de l'émergence du nerf facial lui-même.

\section{$\left.4^{\circ}\right)$ Noyau masticateur.}

C'est le noyau moteur du trijumeau. Il est généralement classé parmi les structures de la protubérance annulaire. Mais il est, chez le Bœuf, presque entièrement placé dans le bulbe rachidien.

Il est formé de cellules multipolaires grandes ou moyennes, qui ressemblent beaucoup à celles du noyau du facial. Ces cellules forment un semis relativement dense dans lequel ne se manifeste pas de subdivision nette. Toutefois, au niveau du quart oral, s'ébauche une division en deux groupes de forme ovalaire, l'un dorsal, le plus volumineux, et l'autre latéro-ventral, voisin de l'émergence de la racine motrice. Cette division s'efface à nouveau à l'extrémité orale du noyau.

Relativement court mais fort épais, le noyau masticateur a une forme générale ovoïde. Son extrémité caudale, située oralement et à peu de distance du noyau du facial débute sur la coupe 3950. Elle est placée un peu oralement au niveau du noyau vestibulaire supérieur, médialement au noyau gélatineux, dorsalement à l'olive supérieure. Sa surface s'accroît rapidement sur les coupes plus orales et il dessine bientôt une large zone ovalaire, ventralement au pédoncule cérébelleux antérieur et au noyau mésencéphalique du trijumeau. Les fibres qu'il fournit se regroupent à son bord 
externe, où elles forment une bande blanche très nette, qui les sépare du noyau sensitif et se prolonge ventro-latéralement pour former la racine motrice du trijumeau. L'extrémité orale correspond à la coupe 4580 , qui appartient à la bordure protubérantielle.

\section{VII. - Formations grises n'appartenant pas aux nerfs crâniens}

Il nous reste à étudier sous ce titre : les noyaux de relais du cordon dorsal de la moelle (Noyaux de Goll, de Burdach et de von Monakow), le noyau du cordon latéral et les noyaux du corps restiforme, l'olive inférieure ou bulbaire, l'olive supérieure ou protubérantielle et enfin les noyaux réticulaires.

\section{Io) Noyaux du cordon dorsal.}

Placés presque entièrement dans la partie post-calamique du bulbe, dont ils occupent la région dorso-médiale, à la terminaison du cordon dorsal de la moelle, ces noyaux sont au nombre de trois :

a) Noyau gracile ou de Goll. - Relativement réduit chez les Ongulés, ce noyau est pourtant mieux individualisé chez le Bœuf que le noyau de Burdach. Il commence dès la partie orale du premier segment cervical et s'étend jusqu'à petite distance de la région calamique. Il est dans tout son trajet placé très près du plan médian, dorsalement au canal de l'épendyme, au sein même du faisceau gracile.

Les cellules qui le constituent, éparses au sein d'une faible nappe névroglique, sont de types divers. Quelques-unes sont multipolaires, de taille moyenne, d'autres, plus nombreuses, ovoïdes ou fusiformes, de taille un peu plus faible, les plus nombreuses enfin, arrondies et petites.

A son extrémité caudale, ce noyau apparaît comme une pointe allongée, portée dorsalement par la portion de la corne dorsale la plus voisine du plan médian. Plus oralement, il s'isole en une masse elliptique, comprimée d'un côté à l'autre, au sein du faisceau gracile. Il semble disparaître au voisinage de la coupe 500, mais plus oralement encore, on en retrouve des éléments diffus, qui peuvent être suivis jusque près de la coupe Iooo.

Ajoutons que sur plus de sa moitié caudale, il est accompagné sur le plan médian par un noyau impair peu développé qui s'élève au bord dorsal de la substance grise sus épendymaire dans la base du septum médio-dorsal.

b) Noyau cunéiforme médial ou de Burdach. - Ce noyau est peut développé et mal différencié chez le Bœuf, où WELEN'SO a montré que son développement s'arrête tôt dans la vie embryonnaire et même est suivi d'une régression.

D'une structure comparable à celle đu précédent, ce noyau apparaît en dehors de celui-ci, dès la partie orale du premier segment cervical, comme une saillie irrégulière et épaisse, portée dorsalement par la base de la corne dorsale. Il grandit rapidement, sans se séparer totalement de la masse grise sous-jacente, et prend une forme très irrégulière, comme déchiquetée. Oralement à la coupe 500 , il devient un peu plus médial, au-dessus du noyau du faisceau solitaire. Il disparaît au voisinage de la coupe I200, sensiblement au niveau du bec du calamus.

c) Noyau cunéiforme latéral ou de von Monakov. - Nettement plus volumineux que les précédents, ce noyau, de forme également très irrégulière, présente des cellules multipolaires plus grandes et plus nombreuses. 
Il commence à s'individualiser au voisinage de la coupe I5O, au־bord latéral du précédent, dont il semble être une dépendance. Il est d'abord mal séparé. Puis il s'élargit oralement et constitue vers le niveau de la coupe goo une masse épaisse, très irrégulière, au bord latéral de laquelle s'enclavent des faisceaux de fibres très myélinisées, qui dépendent manifestement du système vestibulaire descendant. A partir de la coupe I200, il se réduit progressivement, mais, toujours mal délimité, entre en contact dorsalement avec le noyau vestibulaire descendant. Son extrémité orale se place ventralement, entre ce dernier en dehors et la terminaison du noyau triangulaire en dedans. Elle est largement unie à ces noyaux et devient indiscernable aux environs de la coupe 1650 .

\section{$\left.2^{\circ}\right)$ Noyaux $d u$ corps restiforme.}

Ce sont des noyaux multiples et très petits, constitués par de faibles groupes cellulaires répartis à l'intérieur du corps restiforme, non loin de la surface latérale du bulbe. Il s'agit probablement de relais des faisceaux cérébelleux et leur topographie ne peut être précisée.

\section{$\left.3^{\circ}\right)$ Noyaux $d u$ cordon latéral (ou latéraux du bulbe).}

Il s'agit d'une formation particulièrement développée dans l'espèce bovine et nettement subdivisée en deux masses distinctes, l'une médiale et l'autre latérale.

Les deux parties sont caractérisées par leurs cellules multipolaires ou arrondies, de taille moyenne, au sein d'un réseau abondant de fibres peu myélinisées. Le groupe médial est beaucoup mieux délimité que le latéral ; les cellules y sont plus nombreuses et elles deviennent plus grandes dans sa partie orale. De structure plus uniforme, il est ovalaire ou arrondi sur les coupes transversales. Le groupe latéral est moins riche en cellules. Il est aussi beaucoup moins bien délimité, fragmenté en traînées irrégulières dissociées par des paquets de fibres longitudinales et bien myélinisées de la substance blanche bulbaire.

Les deux parties du noyau du cordon latéral s'étendent parallèlement l'une à l'autre, sensiblement sur la même longueur, le noyau latéral étant toutefois un peu plus court. Presque partout bien distincts l'un de l'autre, ils sont cependant en contact ou en continuité à plusieurs niveaux.

L,e noyau médial est placé latéralement à l'émergence du nerf grand hypoglosse et à l'olive bulbaire, à faible distance de la surface ventrale du bulbe. Son extrémité caudale apparaît caudalement à celle de l'olive bulbaire, aux environs de la coupe 600 . Son extrémité orale est située en regard de l'union du tiers moyen et du tiers oral de l'olive. Il se rétrécit à partir de la coupe i7oo et s'étire jusqu'à la coupe Ig8o.

Le groupe latéral commence caudalement à peu près au même niveau que l'olive, soit vers la coupe 800 . Son extrémité orale disparaît aux environs de la coupe I850. Dans toute cette étendue il se montre aplati dorso-ventralement, étiré parallèlement à la surface ventrale du bulbe, ventralement et un peu médialement à la racine descendante du trijumeau.

\section{$\left.4^{\circ}\right)$ Olive bulbaire ou caudale.}

Le complexe formé par l'olive bulbaire et ses noyaux accessoires s'étend sur la moitié caudale de la partie précalamique du bulbe. Sa structure (cellules arrondies 
ou multipolaires, de taille moyenne ou petite ; riche feutrage amyélinique et névroglique) et plus encore, sa situation et son dessin particulier sur les coupes le rendent très facilement reconnaissable. Il est sur toute sa longueur placé non loin du plan médian, dorsalement à la pyramide et médialement aux fibres du nerf grand hypoglosse.

L'olive principale se présente sur la plus grande part de son étendue comme une sorte de bourse aplatie dorso-ventralement et ouverte par un hile du côté médial et un peu dorsalement. Elle se trouve ainsi subdivisée en deux lamelles, l'une ventrale et l'autre dorsale, cette dernière un peu plus faible et plus courte. Cette disposition s'efface aux extrémités, surtout caudale, où l'olive apparaît comme un noyau plus mal délimité mais dépourvu de hile. L'extrémité caudale correspond à la coupe 960, et l'extrémité orale à la coupe 2720 .

L'olive accessoire médiale ou médio-ventrale est la mieux développée. Épaisse et relativement importante, elle est placée ventralement. Son extrémité caudale apparaît, à peu près au niveau de celle de l'olive principale, comme une lame aplatie et large, très étalée sous le noyau olivaire principal, qui lui est accolé. Plus oralement, vers la coupe 1360 , elle devient complètement isolée. Elle se rétrécit progressivement mais devient beaucoup plus épaisse. Elle disparaît assez brusquement au voisinage de la coupe 2200 .

L'olive accessoire dorsale est beaucoup plus faible, comme d'ailleurs la lamelle dorsale de l'olive principale, dont elle n'est bien séparée que dans sa partie moyenne. Elle ne commence à être discernable que vers la coupe r20o ; c'est alors une masse irrégulière, mal délimitée, portée en quelque sorte par la lamelle dorsale. Elle s'isole ensuite en une petite plaque étroite (coupes I 800 à 2200) puis s'étale, devient comme diffuse et s'unit à nouveau à la lamelle dorsale. Elle devient indiscernable au niveau de la coupe I520.

\section{$\left.5^{\circ}\right)$ Olive protubérantielle ou rostrale.}

C'est une masse grise volumineuse qui siège à la partie ventrale de la jonction bulbo-protubérantielle, médialement au noyau du facial. Chez le Bœuf,, la partie bulbaire en est bien plus étenđue que la partie pontique. Il s'agit en fait d'un véritable complexe, complètement subdivisé en deux noyaux, l'un ventro-médial et 1'autre dorso-latéral, ce dernier beaucoup plus court. Tous deux sont formés de cellules de taille moyenne ou petite, de forme plus ou moins sphérique, au sein d'un réseau fibrillaire dense.

L'extrémité caudale des deux noyaux apparaît presque au même niveau, au voisinage de la coupe 3600 . Elle est placée entre les fibres du nerf moteur oculaire externe et la partie orale du noyau du facial. Chaque noyau présente une section arrondie et devient rapidement très volumineux. Il est délimité par une couche fibrillaire serrée et pénétré par des faisceaux de fibres. Ces dernières dessinent, sur les coupes transversales du noyau dorso-latéral un $\mathrm{S}$ et, plus oralement, un 8 , qui marque la division de ce noyau en deux groupes cellulaires, 1'un dorsal et l'autre ventral. Le réseau fibrillaire périphérique est uni aux noyaux acoustiques par dé nombreux faisceaux de fibres, sur le trajet desquels sont placés de multiples petits groupes cellulaires. Le noyau dorso-latéral disparaît à la jonction bulbo-pontique (coupe 4IOo), et seul se poursuit dans la protubérance le noyau médio-ventral, médialement au noyau masticateur. 


\section{0) Formation réticulée.}

Fort bien développée chez le Bœuf, la substance réticulée est surtout caractérisée par le grand volume de ses cellules. Elle constitue, comme dans les autres espèces, différents amas ou noyaux parmi lesquels il est aisé de reconnaître :

a) le noyau du raphé, étendu dans le plan sagittal et mieux développé à certains niveaux qu'à d'autres ;

b) le noyau du corps trapézoïde, placé médialement à l'extrémité caudale de l'olive protubérantielle et prolongé par une traînée cellulaire jusqu'entre les fibres du corps trapézoïde.

c) les noyaux arqués, disséminés au voisinage de la face ventrale du bulbe.

\section{CONCLUSION}

La relative précision des descriptions qui précèđent et des illustrations qui les matérialisent ne saurait masquer les doutes qui nous ont bien souvent assaillis au cours de leur élaboration.

En premier lieu, les limites topographiques indiquées, et en particulier les niveaux caractérisés par les numéros de coupes, ne doivent pas être considérés comme des résultats absolus. L,eur valeur ne peut être qu'indicative. Il est d'abord évident que les coupes histologiques ne peuvent pas être absolument perpendiculaires au plan médian. C'est ainsi qu'en examinant les deux côtés d'une même coupe, on ne trouve jamais exactement les mêmes détails structuraux. Pour établir notre numération, nous avons dû ne tenir compte que d'un seul côté des coupes, toujours le même. D'autre part, la limite des noyaux est rarement assez précise pour qu'elle puisse être située sur une seule coupe. Force est donc de retenir un chiffre seulement approximatif. Enfin, il existe indiscutablement de légères variations volumétriques d'un côté à l'autre et très certainement des variations plus importantes encore d'un individu à l'autre. C'est pourquoi le présent travail ne peut être qu'un élément de base pour de nouvelles recherches.

D'autre part, si certains noyaux sont reconnaissables sans aucune hésitation et faciles à délimiter, il n'en est pas de même pour toutes les structures bulbaires. Nous avons déjà exposé nos doutes relatifs aux noyaux salivaires.

L'identification et surtout la délimitation d'autres éléments tels que le noyau gustatif ou certains des noyaux vestibulaires posent des problèmes du même ordre. Dans ce domaine encore, le présent travail appelle de larges contrôles soit embryologiques, soit expérimentaux. Une étude actuellement en cours de différentes lésions provoquées chirurgicalement chez le Veau permettra peut-être de préciser les connexions et la valeur fonctionnelle de divers noyaux.

Il n'en reste pas moins que les résultats déjà acquis permettent les remarques suivantes :

- Certains noyaux présentent chez le Bœuf un grand développement. Ce sont en particulier : le noyau du nerf grand hypoglosse, épais et complexe mais pourtant moins avancé oralement que chez les Équidés et la plupart des autres espèces, le noyau intercalaire de Staderini, le noyau dorsal du pneumogastrique, le noyau ambigu, le noyau du facial, l'olive protubérantielle et les noyaux du cordon latéral. 
Par contre, le noyau gustatif et les noyaux salivaires sont fort mal individualisés. Les noyaux vestibulaires sont très mal délimités et le noyau dorso-médial de Schwalbe n'est pas distinct.

Ia particularité topographique la plus remarquable concerne la jonction bulboprotubérantielle. La protubérance annulaire s'étend beaucoup moins caudalement que chez la plupart des autres Mammifères. Elle laisse ainsi à découvert une plus grande partie du corps trapézoïde, qui paraît très large. Cette disposition a pour conséquence de transférer à la partie orale du bulbe des formations dont la topographie est nettement protubérantielle dans la plupart des autres Mammifères. La situation de l'olive protubérantielle et du noyau masticateur est caractéristique à cet égard.

Reģu pour publicalion en mai 1964 .

\section{SUMMARY}

STUDIES ON THE MORPHOLOGY AND TOPOGRAPHY OF THE GREY MATTER IN THE MEDULLA OBLONGATA IN THE COW

The morphological and structural characters of the grey matter and the topography of the nuclei in it were studied on serial sections of the medulla oblongata of a heifer of 18 months. A graphical reconstruction was made of the principal nuclei.

The nucleus of the hypoglossal nerve, the nucleus intercalatus, the dorsal nucleus of the vagus, the nucleus ambiguus, the facial nucleus, the oral oliva and the nuclei laterales were seen to be strongly developed. The gustatory and salivary nuclei and certain vestibular nuclei were not well defined. The junction of medulla and pons is more rostral than in most other mammals and this causes an extension of the medullary region in the same direction.

\section{RÉFÉRENCES BIBI,IOGRAPHIQUES}

Anoroso E. C., Beil F. R., RosenberG H., 1952. The localization of respiratory regions in the rhombencephalon of the sheep. Proc. Roy. Soc., B 139, 128-140.

Aniderson B., Kitchell R., Person N., i958. A study of central regulation of rumination and reticuloruminal motility. Acta Physiol. Scand, 46, 319-338.

Barone R., 1962. Anatomie des Équidés. Tome III, fascicule I. Névraxe et Méninges, Lyon, ig62.

Barone R., Duroux, Bossy. Éléments d'anatomie comparée de l'isthme encéphalique chez quelques Mammifères. C. R. Assoc. Anat., 1955, 42, 244-254.

BeLl F. R., 1960. The localization within the dorsal motor nucleus of the vagus of the eff erent fibres to the ruminant stomach. J. Anat., 94, 410-417.

BeLl F. R., LAwN A. M., I955. Localization of regions in the medulla oblongata of sheep associated with rumination. $J$. Physiol., London, 128, 577-592.

Cajal Ramon S., rgog. Histologie du système nerveux de l'Homme et des Vertébrés. Tome I, Maloine, Paris.

Chomik M., 1952. Motor nuclei of the medulla oblongata in the cow, pig and horse (en polonais). Ann. Univ. M. Curie Sklodowska Lublin, Sect., C 5, 373-440.

Chomiak M., 1952. Der Kern des Seitenstranges (nucleus funiculi lateralis) bei der Kuh, dem Schwein und Pfred. (En polonais). Ann. Univ. M. Curie Sklod. Lublin, Sect. C 6, 14-24.

CHomik M., r953. Topography and structure of the motor nuclei of the medulla oblongata in the goat. (En polonais.) Ann. Univ. M. Curie Sklod., Lublin, Sect DD, 8, 181-219.

Dejerine, 190I. Anatomie des centres nerveux. Tome II, Rueff, Paris.

Dussardier M., ig60. Recherches sur le contrôle bulbaire de la motricité gastrique des Ruminants. Thèse Doc. Sciences, Paris.

Hoffmany G., I 955. Topographischer und zytologischer Atlas der medulla oblongata von Schwein und Hund. Disch. Akad. Landw. Wiss., Berlin, 8, I-168. 
Jornston J. B., igo9. The radix mesencephalica trigemini. J. Comp. Neurol, 19, 593-644.

Kappers C. U. Ariens, Huber C., Crosby E. C., ig6o. The comparative analomy of the nervous system of vortebrates, including man. Hafner publishing Comp., New York.

Kappers C. U. Ariens, 1946. Anatomie comparée du système neveux. Masson, Paris-Amsterdam.

Kitcifel, R. L., Stromberg M., Davis L. H., i956. Comparative studies of the dorsal vagal motor nucleus in ruminants and non ruminants. Amer. Assoc. Anat. 1956, in Anat. Record., 124, 319.

LEWINJOWSKA M., I953. Nucleus fasciculi gracilis and nucleus fasciculi cuneati in the domestic ruminants. (En polonais.) Ann. Univ. M. Curie Sklod., Lublin Sect. DD8, 339-352.

Lombard C., 1957. Contribution à l'étude des noyaux branchio-moteurs des nerfs crâniens. Thèse Doc. Med., Lyon.

Marsland T., Glies P., Erikson L. B., i 954. Modification of the Glees silver impregnation for parafin sections. 7. Neuropath. exp. Neurol., 13, 587.

Palmer A. C., 1958. Anatomical arrangement of the grey matter in the madulla, pons and midbrain of the sheep. Zbl. Vet. Med., 5, 953-967.

Pattison I. H., Holman H. H., 1943. A guide to the internal structure of the medulla oblongala of the sheep. J. Comp. Palhol. Therap., 53, г $3^{\mathrm{I}-\mathrm{I}} 39$.

Rucinski T., Welento J., 1956. Development of posterior olive in cattle. (En polonais.) Ann. Univ. M. Curie, Sklod. Lublin, Sect. DD 11, I47-162.

Stevens C. E., Sellers, I959. Studies of the reflex control of the ruminant stomach with special reference to the eructation reflex. Amer. J. Vet. Res., 20.

Welento J., 1957. The development of the nucleus gracilis NAP and nucleus cuneatus NAP in the cow. (En polonais.) Ann. Univ. M. Curie Sklod. Lublin, Sect. DD12, 65-75.

Welento J., I955. The nuclei of the anterior part of the medulla oblongata and pons of the brain in cattle. (En polonais.) Ann. Univ. M. Curie Sklod. Lublin, Sect. DD, 10, I85-238.

WeElento J., Bobkiewicz A., 1954. The development of the motor nuclei in the medulla oblongata of the cow. (Iin polonais.) Ann. Univ. M. Curie. Sklod. Lublin, Sect. DD, 9, 235-267.

Wixckler G., 1959. A propos de la technique de l'imprégnation argentique de Marsland, Glées et Erikson. Arch. Anat. Hist., 42, 231 . 


\section{ANNEXE (PIANCHES)}
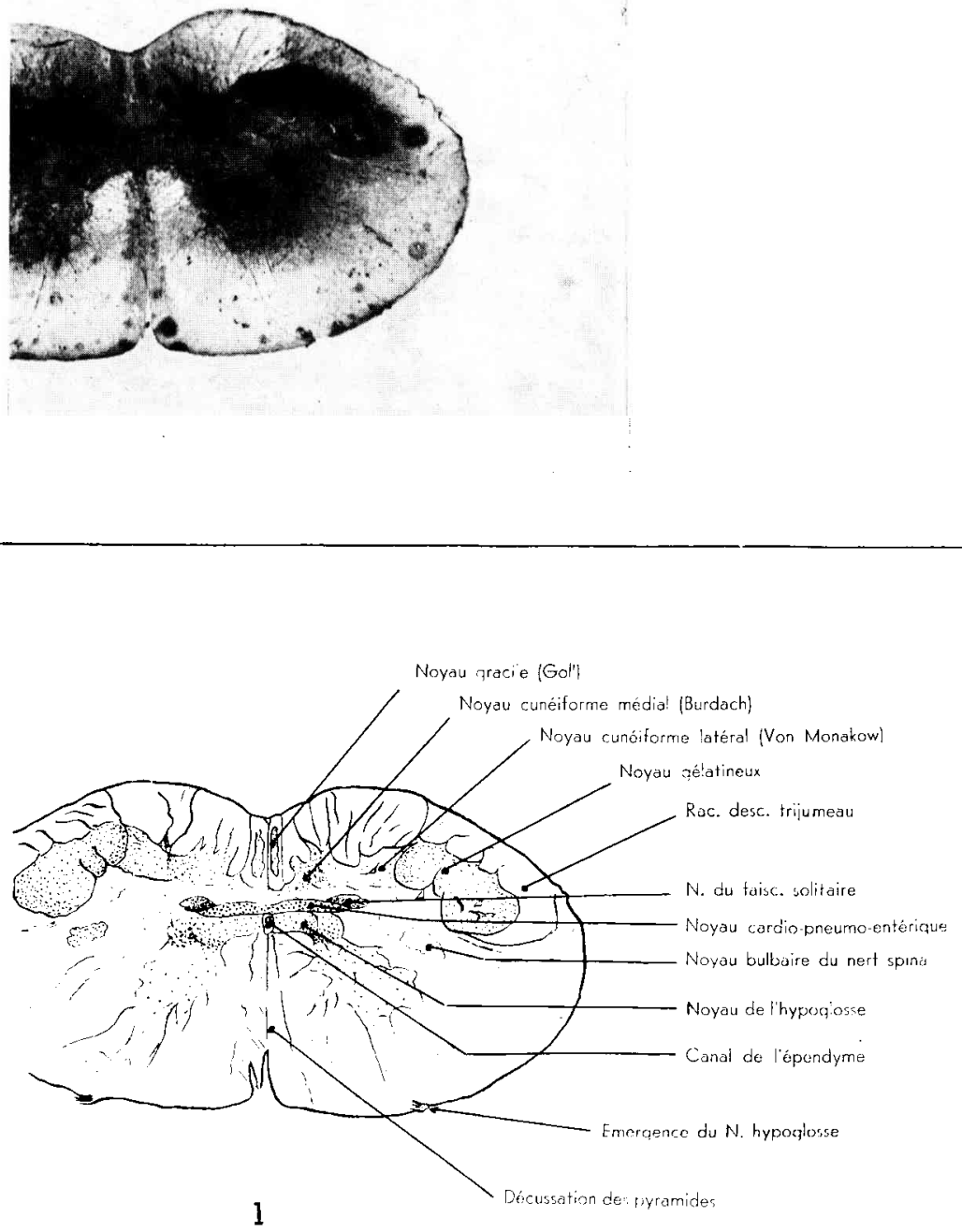

Plancie I.

X. B. Les numćros cles coupes transversales correspondent aux niveaux indiqués sur les planches i i et t 2 . 

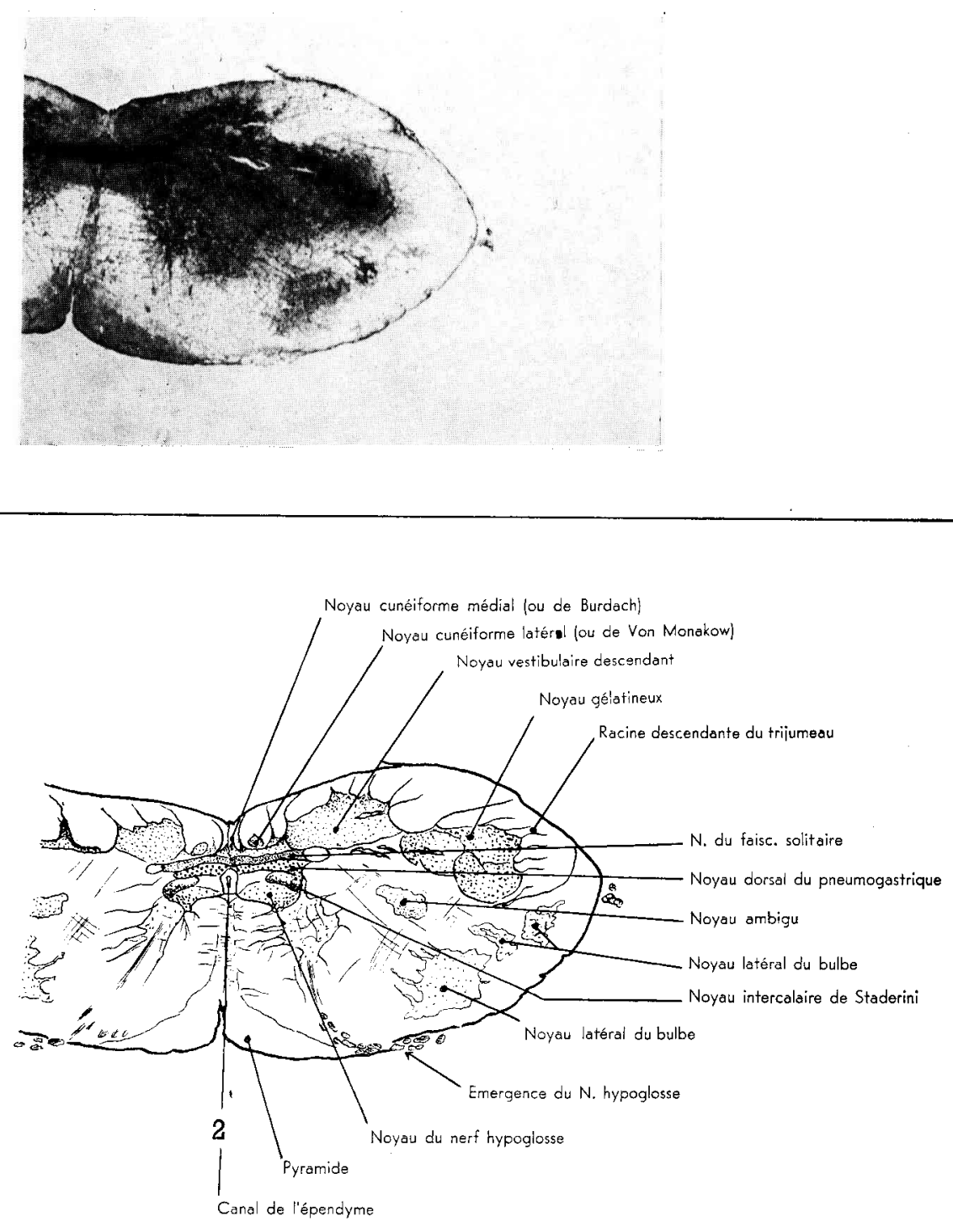

PLANCIE 2. 

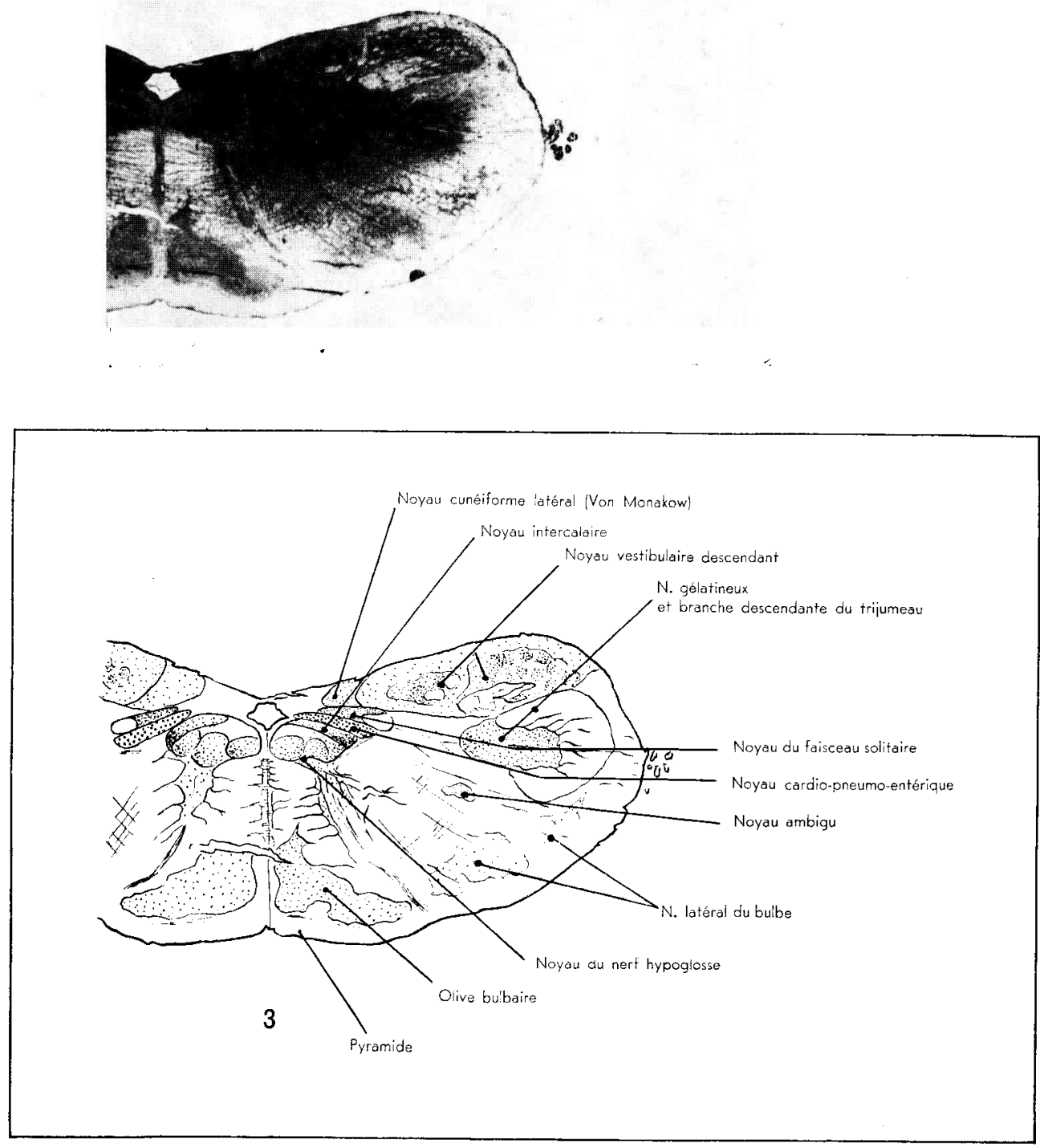

Plinche 3. 

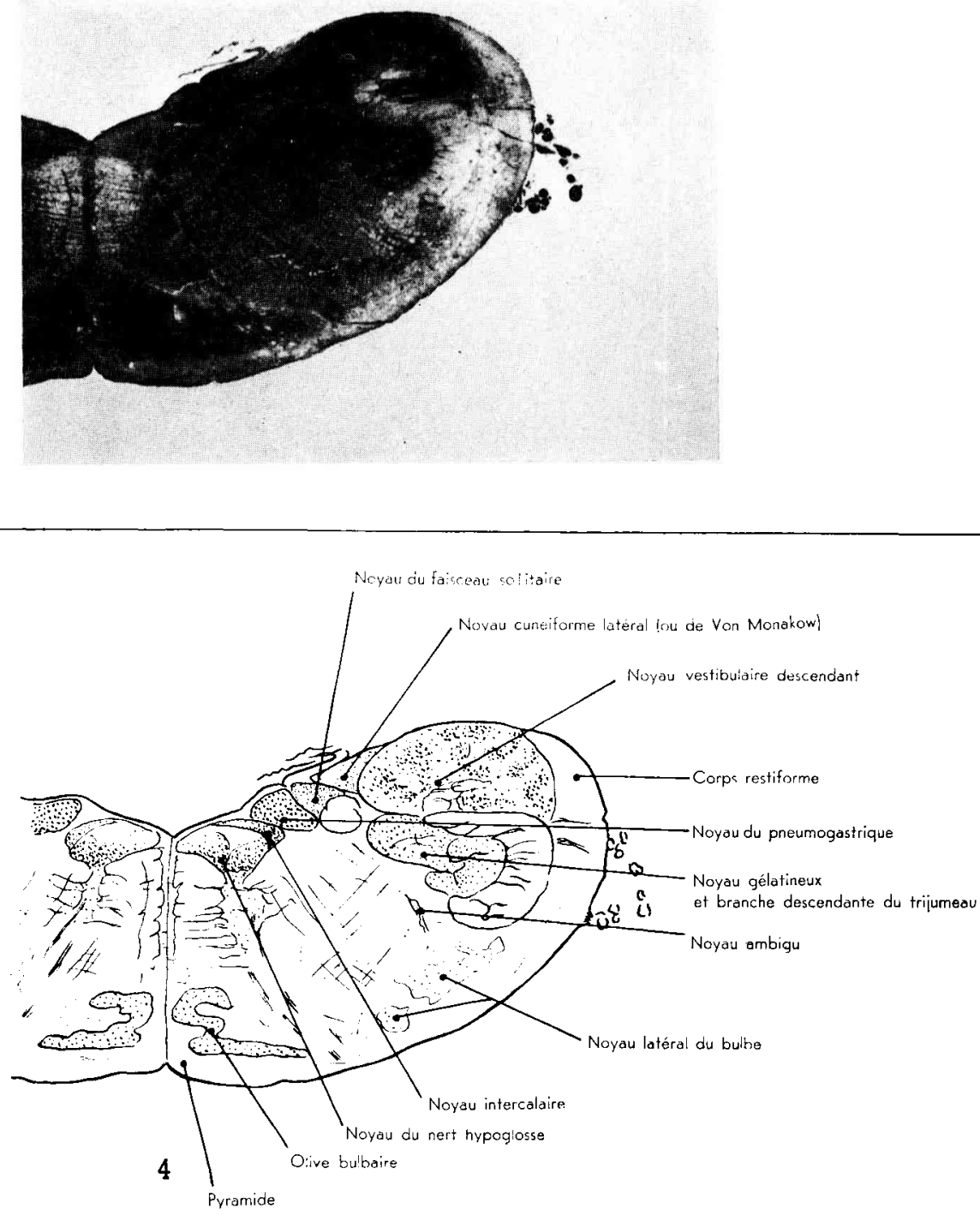

Plavile 4. 

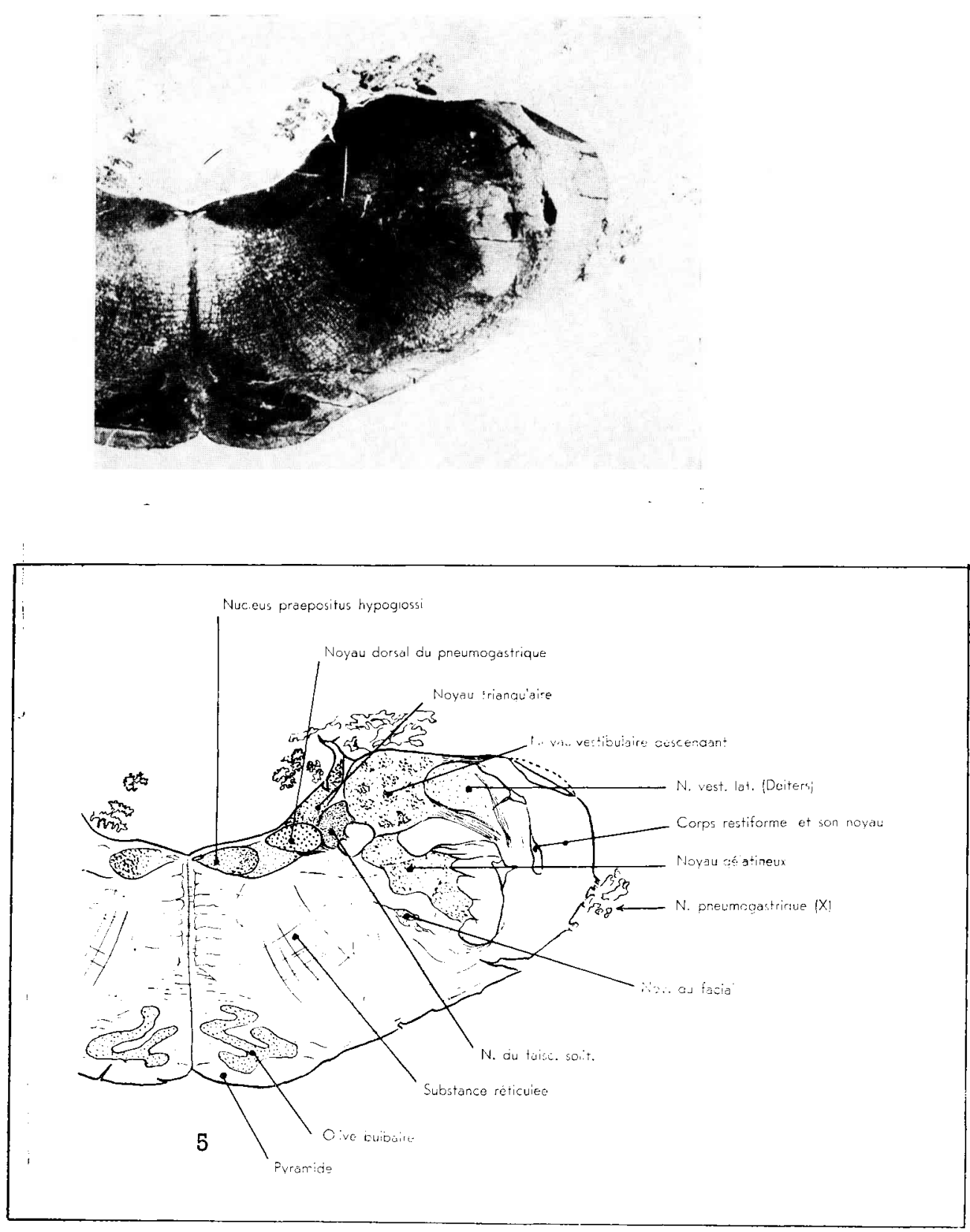

Plancie 5. 

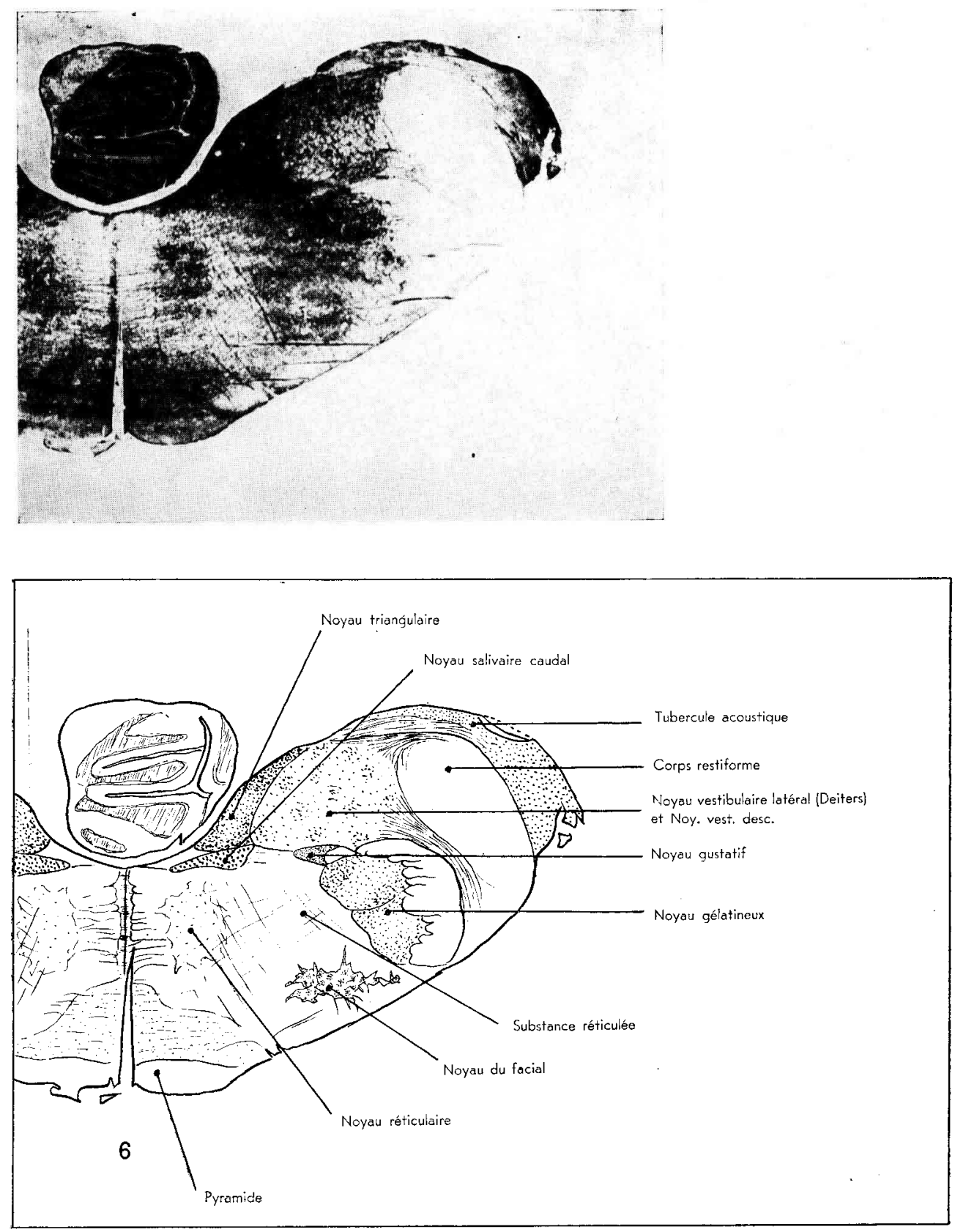

Planche 6. 

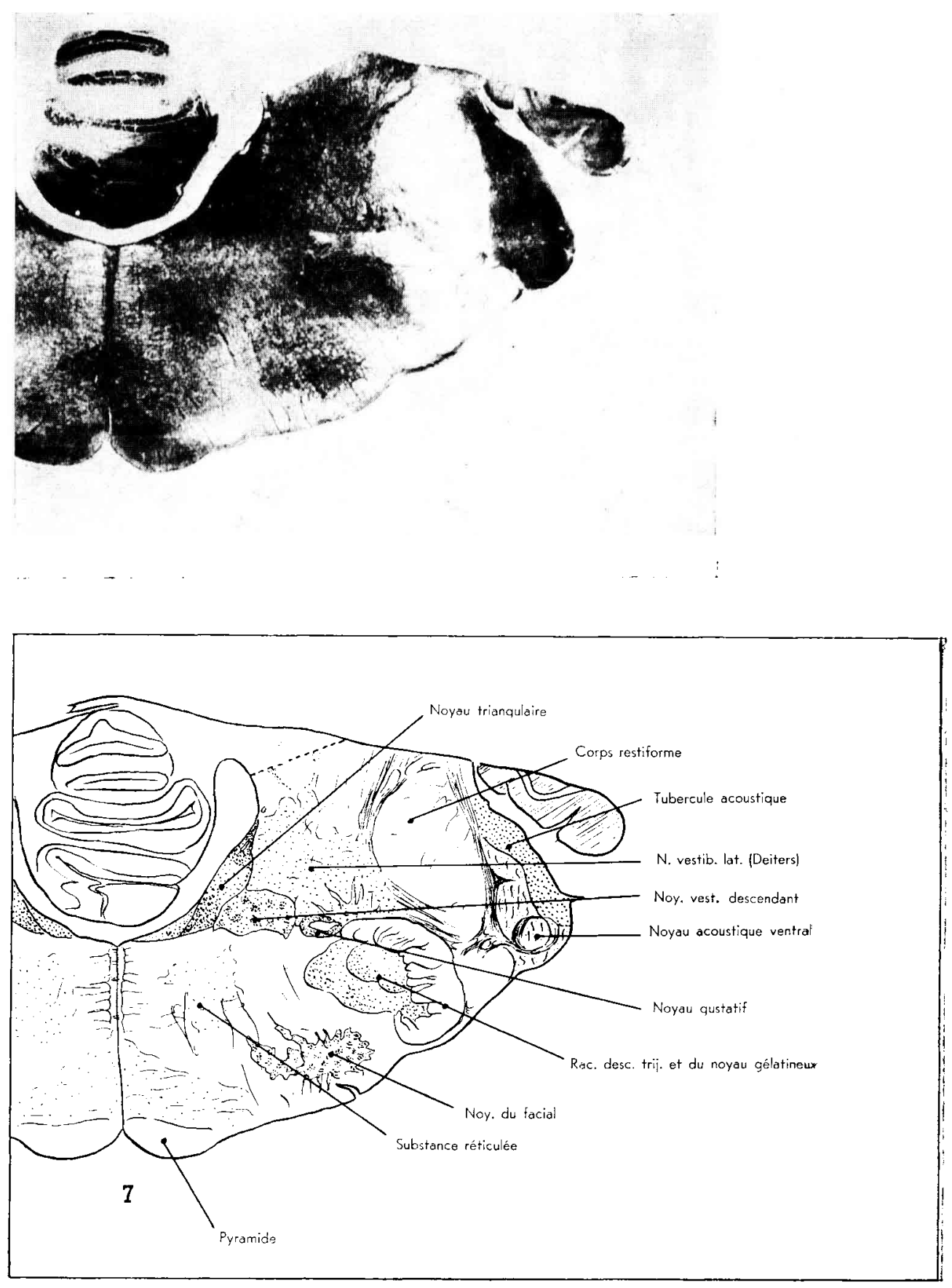

Planciele 7. 

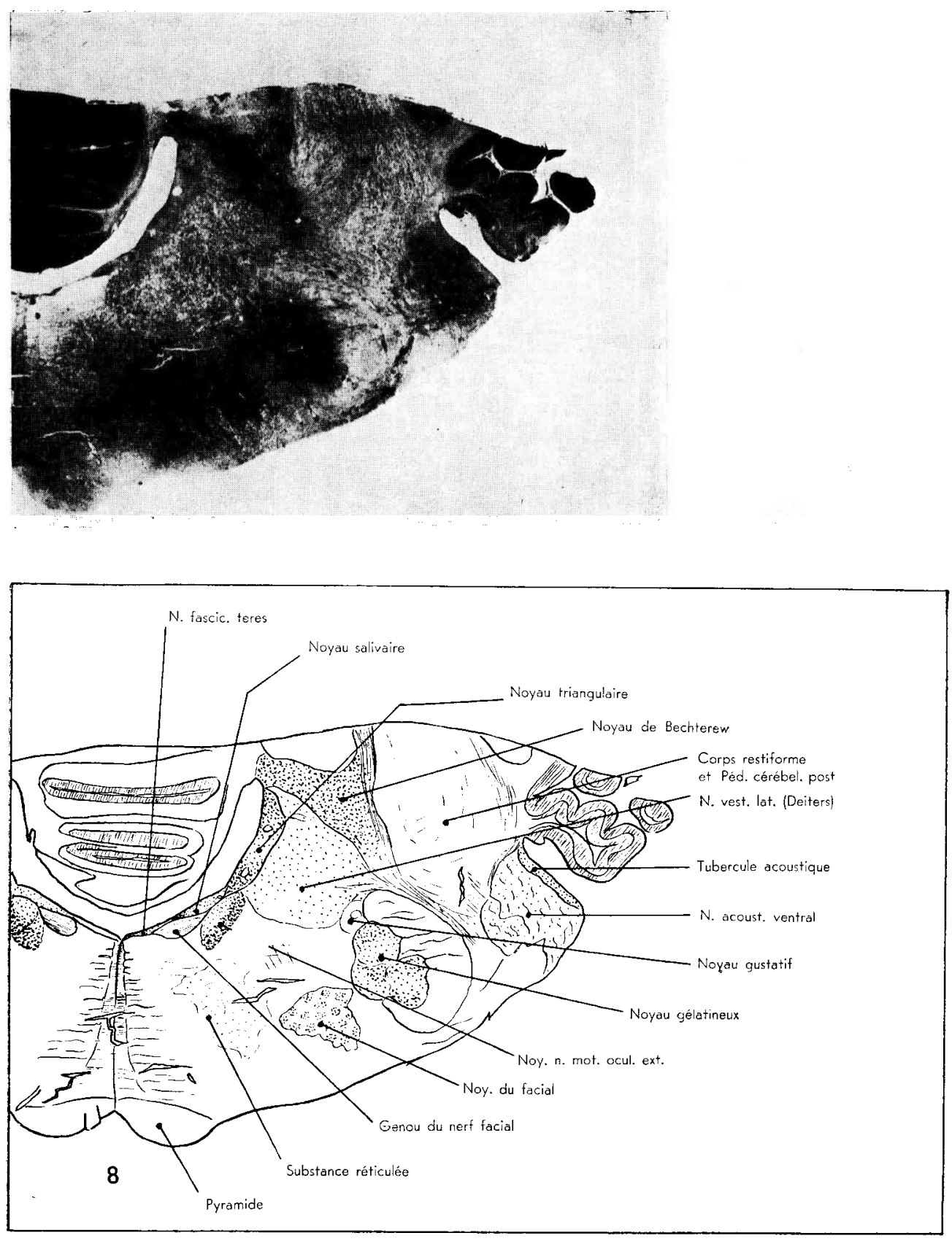

Planche 8. 

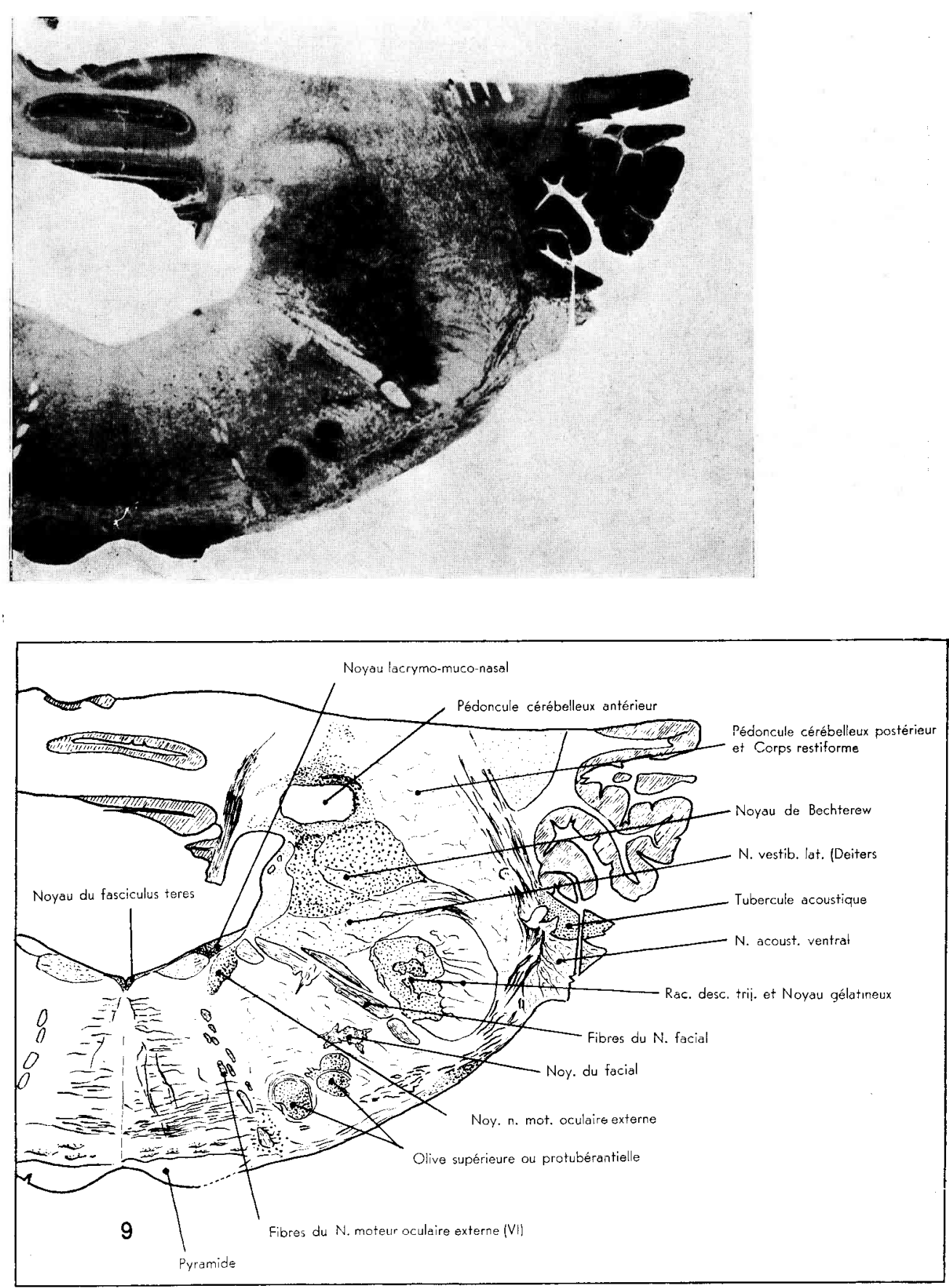

Planche 9 . 

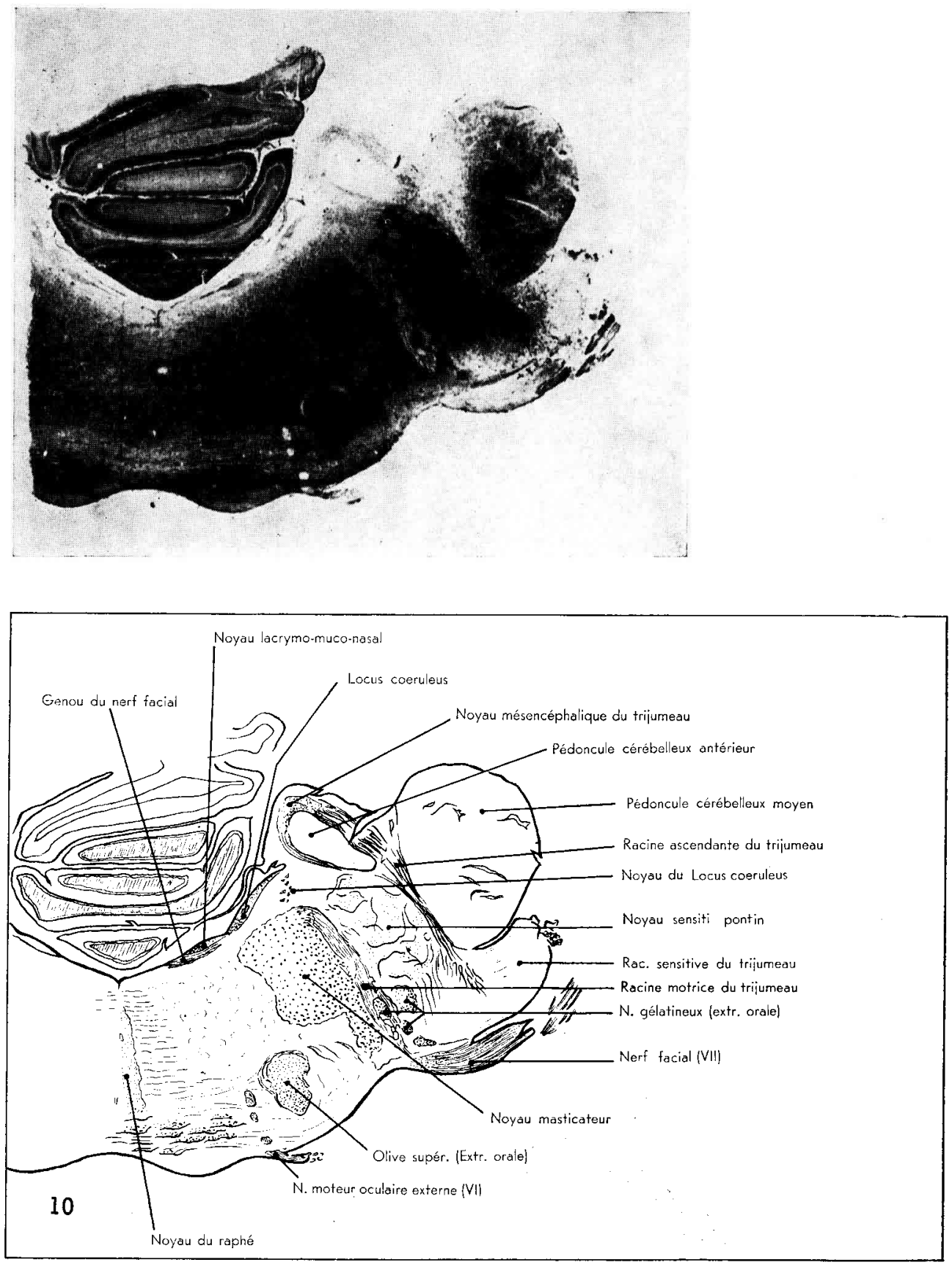

Planche io.

tit 


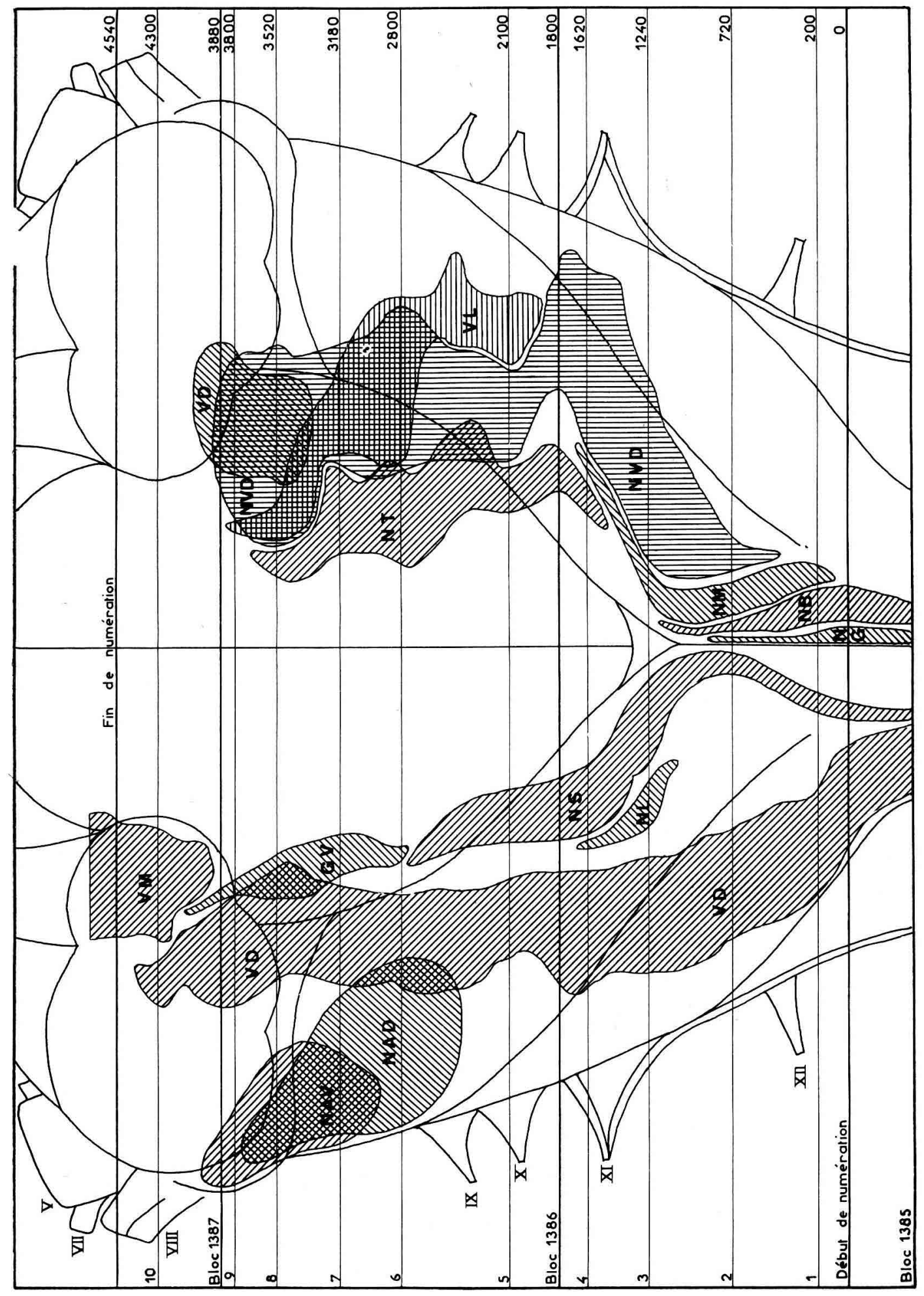




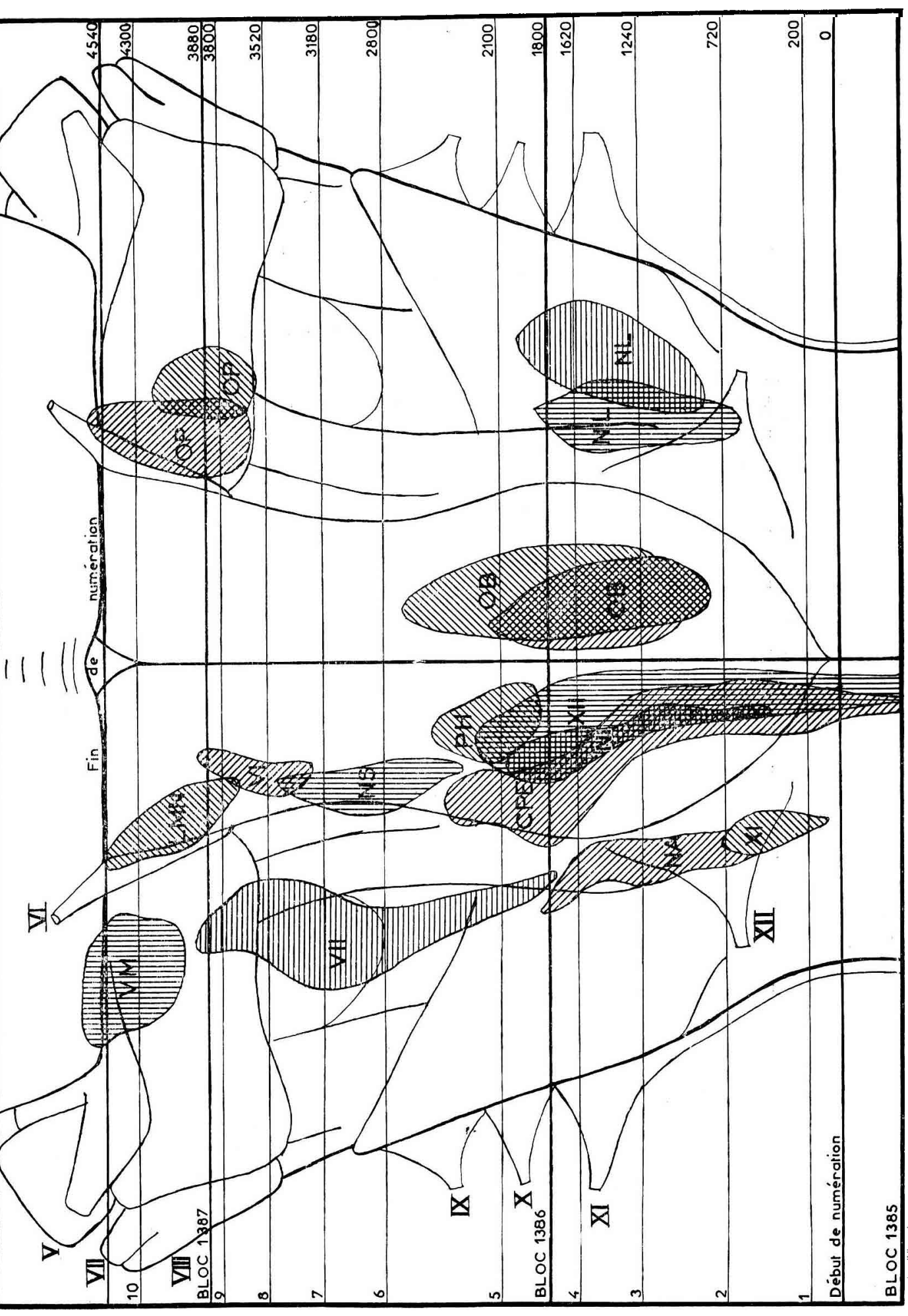

Annales de Biologie animsle. - I 964. 


\section{LEGENIES DES PLANCHES 1 i t 12}

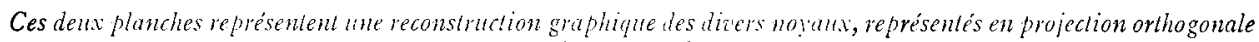
sur la surface bulbare

I'lanche i I, --. I'rojection des noyaux d'afférences sur la face dorsale du bulle:

Côté grauche : Noyaux extéroceptifs et visceroceptits.

VD : Noyau gélatincus ou de la racine descendante du 'Jrijuneau.

Vll : Noyau mésencéphalique du Trijumeau.

NAV : Noyau acoustique ventral.

XID : Novau acoustique dorsal.

IS : Noyau du faisceau solitaire.

NL : Noyau latéral du faisceau solitaire.

GV : Noyau gustatif.

Côté droit : Noyaux proprioceptifs.

$\backslash \mathrm{G}$ : Noyau gracile ou de Goll.

NI : Noyau de Burdach ou cunciforme médial.

NM : Noyau de Von Monakow ou cuncéforme latéral.

NVI : Noyau vestibulaire descendant.

NT: Noyau trianculaire.

$V I$ : Noyau vestilijulaire latéral.

VD : Noyau vestibulaire dorsal.

Planche 12. - l'rojcction des noyaux alefférences et des noyaux propres du bulbe sur lia face zentrale du bulbe

Côté gauche : Noyaux moteurs et sympathiques efférents:

VXI : Noyau masticateur ou noteur du trijuneau.

VII : Noyau du nerf facial.

NA : Noyau anbiru.

XI : Noyau du nerf spinal.

LMIN : Noyau lacrymo-1unce-nasial.

VI : Novau du nerf oculo-moteur externe.

NS : Noymu salivaire.

CPE : Noyau cardio-pnicumo-cuterique on dorad du vasule.

PH : Nucleus praepositus hyporlossi.

$X I$ : Yoyau intercalate de Staderini

XII : Noyau du mitul hypoglosse.

Cóté droit : Noyaux propres du bulbe :

OB : Olive bulbaire ou inférieure (on a représcnté en outre les limites rle l'olive accessoire ventrale).

OP : Olive protuberantielle ou orale (2 novaux).

NL : Noyaux latéraux du bulbe. 


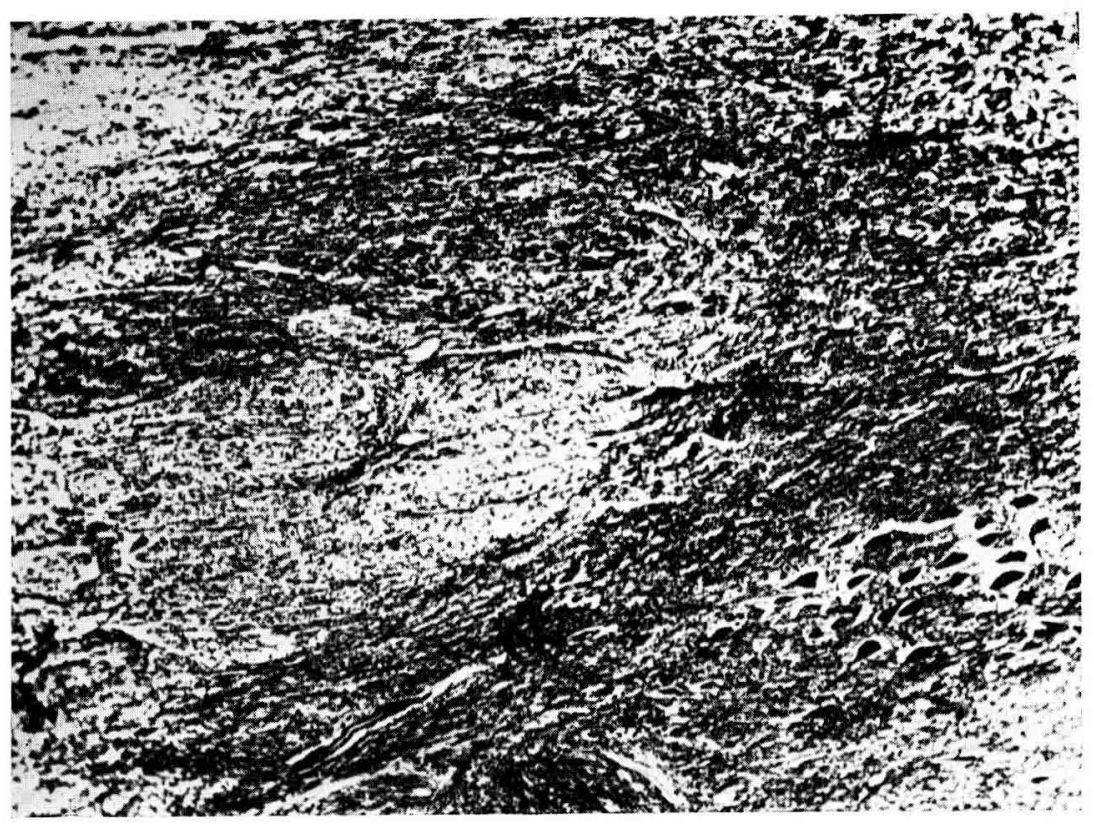

\section{Iraiscean solitaire et son noyau}

Coupe 1240 . Gr $\times 60$

I.e faisceau solitaire (clair, an centre et à ganche) est conme encluvé dans le noyau, qui le bonde en haut et en bas et s'étend jusqu'atu bord droit de la photo. A droite et en biss, sous le noyati du faisceitu solitaire. oft voit apparaitre le noyau dorsal du pneumogastrique, avec ses cellules fusiformes.

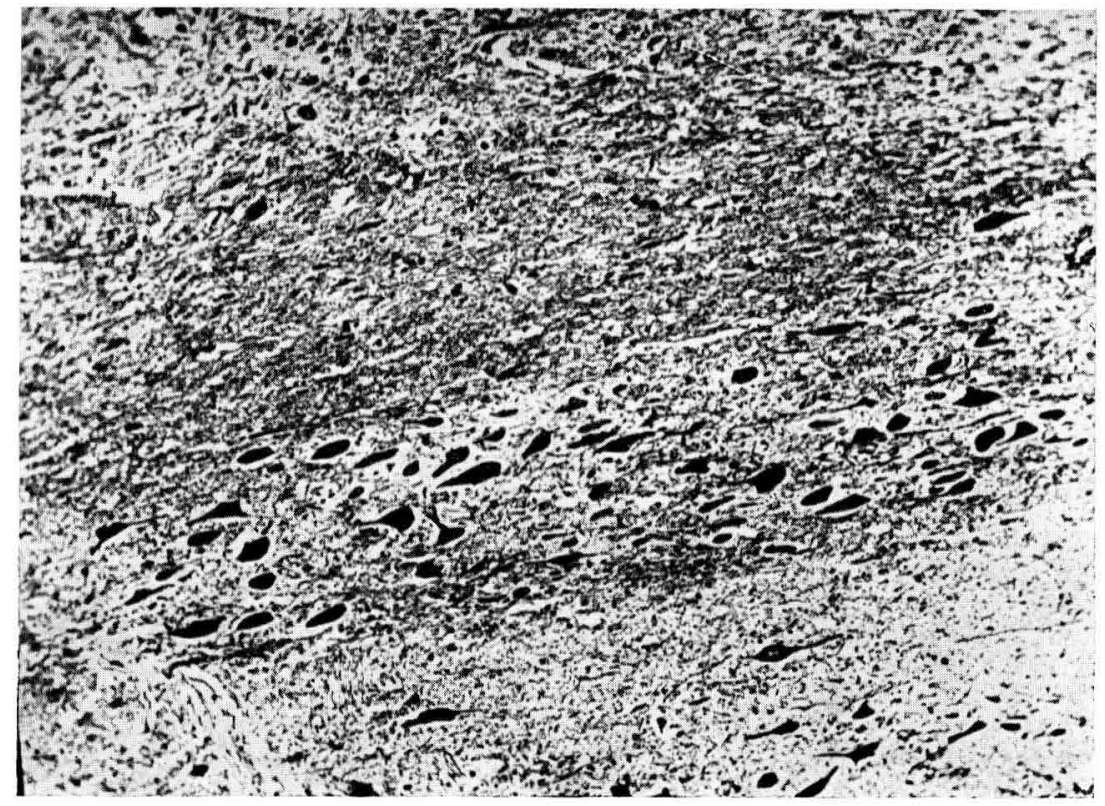

Noyau dorsal du pneumogastrique

Coupe 1230. Gr. $\times 70$

Caractérisé par ses cellules fusiformes, ce noyau est accompagné en haut par le noyau du faisceau solitaire et en bas par le noyau intercalaire. 



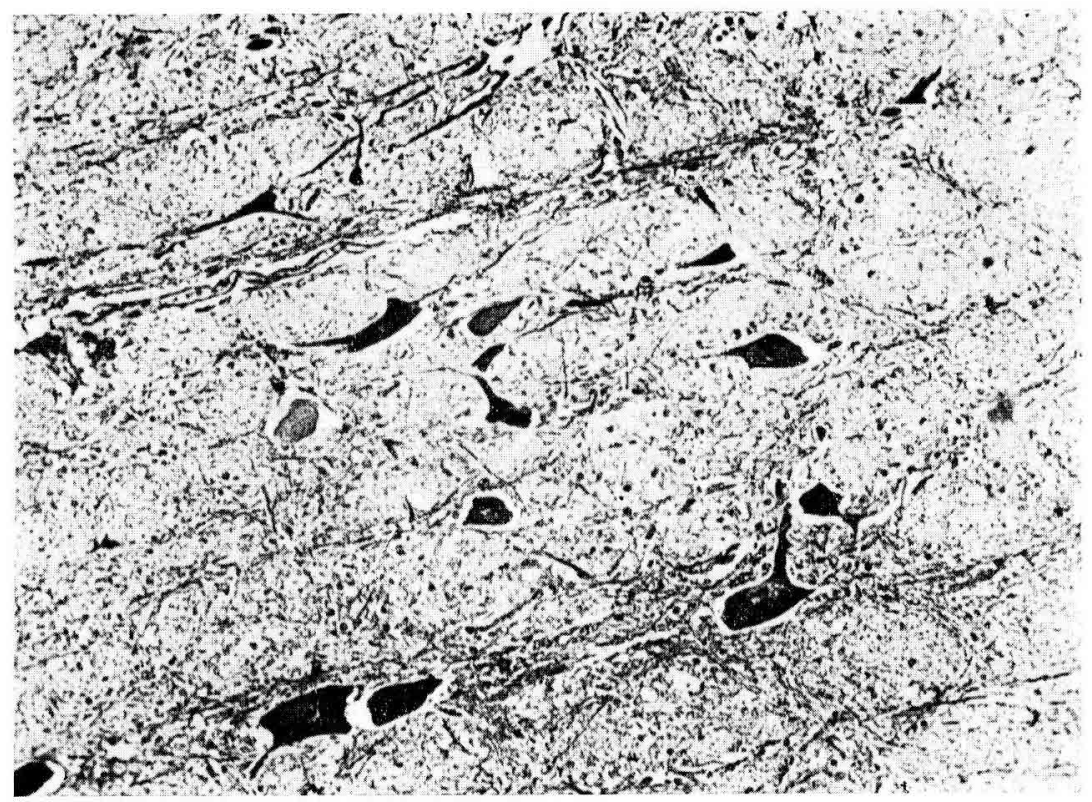

Cellules du noyau ambigu

Coupe 1220. Gr. $\times 90$

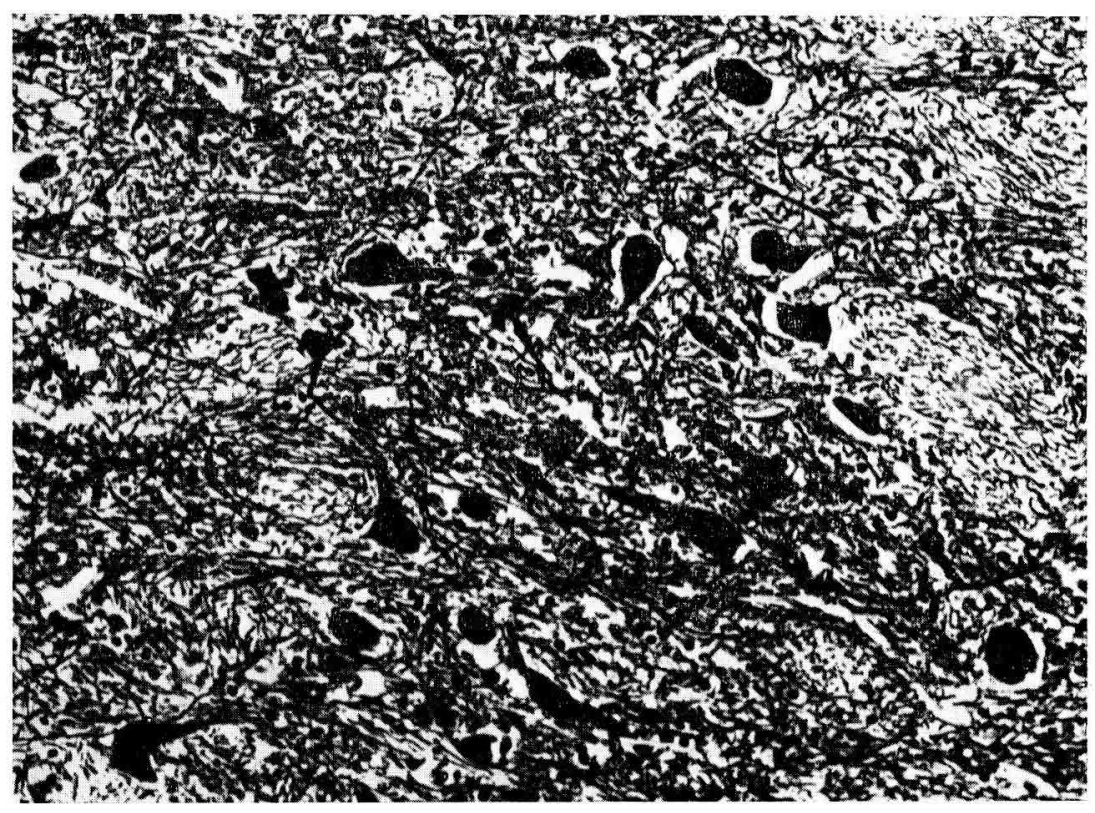

Cellules et faisceand de fibres dn noynu restibulaire descendant 



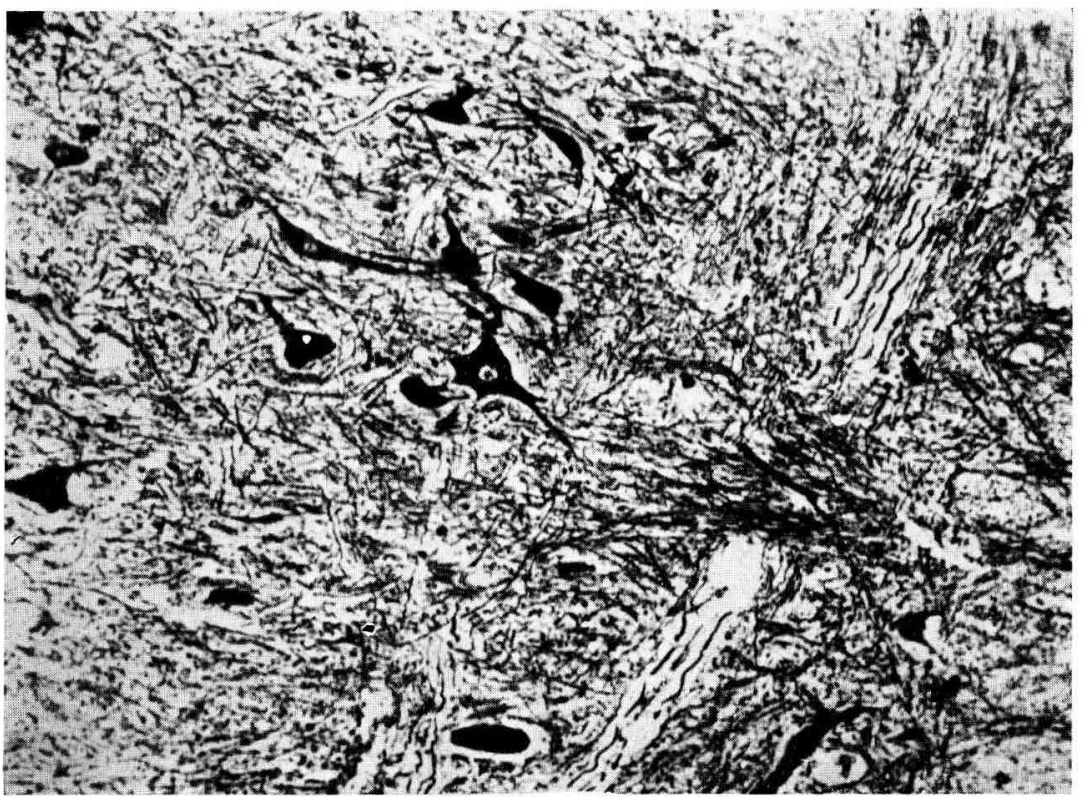

Voyan dorsal da nerf stand hyprolosse

Cirandes cetlules multipolaires átolés

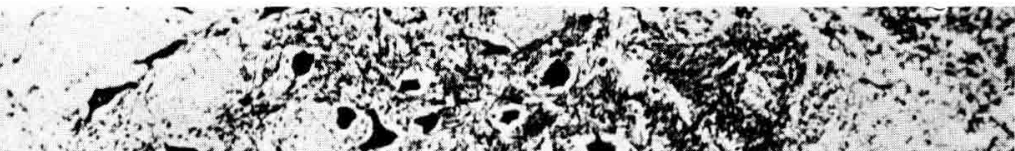

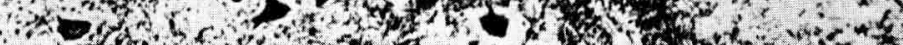

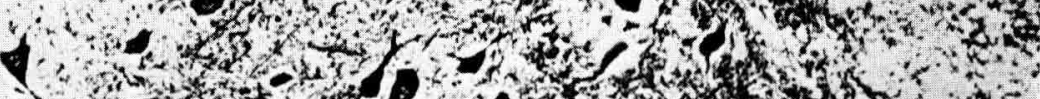

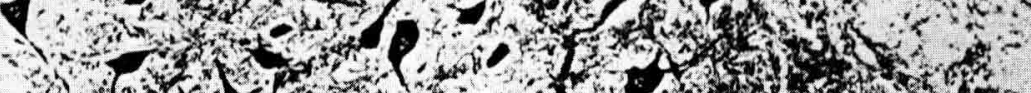
1.

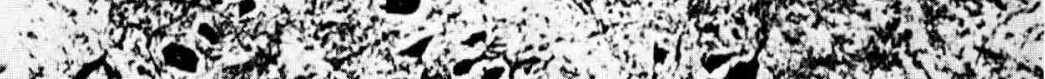
4.

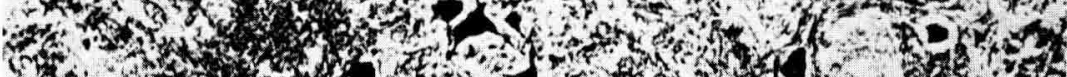
(m) (1. 